\title{
Interval-Valued q-Rung Orthopair Fuzzy QUALIFLEX Decision Analysis Method with Dombi Operators
}

\author{
Benting Wan $\mathbb{D}^{1},{ }^{1}$ Xiaolu Zhang $\mathbb{D}^{2}{ }^{2}$ Meifang Xiong, ${ }^{3}$ and Zhenwu Wang $\mathbb{D}^{4}$ \\ ${ }^{1}$ School of Software and IoT Engineering, Jiangxi University of Finance and Economics, Nanchang 330013, China \\ ${ }^{2}$ The Collaborative Innovation Center, Jiangxi University of Finance and Economics, Nanchang 330013, China \\ ${ }^{3}$ College of Modern Economics \& Management, Jiangxi University of Finance and Economics, Nanchang 330013, China \\ ${ }^{4}$ Department of Computer Science and Technology, China University of Mining and Technology, Beijing 100083, China \\ Correspondence should be addressed to Xiaolu Zhang; xiaolu_jy@163.com
}

Received 11 October 2021; Revised 6 January 2022; Accepted 13 January 2022; Published 22 February 2022

Academic Editor: Marko Robnik

Copyright ( 2022 Benting Wan et al. This is an open access article distributed under the Creative Commons Attribution License, which permits unrestricted use, distribution, and reproduction in any medium, provided the original work is properly cited.

\begin{abstract}
As a new and useful decision tool, interval-valued q-rung orthopair fuzzy set (IVq-ROFS), has strong ability to address uncertain or imprecision information in practical decision-making processes. This paper develops a new interval-valued q-rung orthopair fuzzy QUALIFLEX decision analysis method with Dombi operators for handling the group decision-making problems with IVqROFSs data. We also conduct a case study on the health management of hypertensive patients for demonstrating the feasibility and superiority of the developed method. The main contributions of this paper are as follows: (1) we first propose interval-valued q-rung orthopair fuzzy Dombi operational laws and investigate their desirable properties; (2) using the developed Dombi operational laws, we develop an interval-valued q-rung orthopair fuzzy Dombi weighted average (IVq-ROFDWA) operator, and we prove that the IVq-ROFDWA operator satisfies the properties of idempotency, commutativity, boundedness, and monotonicity; (3) we introduce the concept of interval-valued q-rung orthopair fuzzy ideal group opinions with the help of IVqROFDWA operator and propose a new weight-determining method for identifying the weights of experts; (4) we develop a new interval-valued q-rung orthopair fuzzy distance measure. With the help of the developed distance measure, we calculate intervalvalued q-rung orthopair fuzzy concordance/discordance index under each permutation, and then a new interval-valued q-rung orthopair fuzzy QUALIFLEX decision analysis method is developed for addressing the complex multiattribute group decisionmaking problems with IVq-ROFSs.
\end{abstract}

\section{Introduction}

With the increasing complexity and uncertainty in real-life decision-making processes, many useful extensions of fuzzy sets, such as the classical intuitionistic fuzzy sets (IFSs) [1], Pythagorean fuzzy set (PFS) [2, 3], interval-valued PFS [4], and the generalized orthopair fuzzy set [5], have been developed successively to describe the uncertain estimated results in complex practical multiattribute decision-making (MADM) problems [6]. The generalized orthopair fuzzy set is also called q-rung orthopair fuzzy set and is known simply as q-ROFS in the existing literature. Compared with the IFS and the PFS, the q-ROFS can contain more fuzzy information. In the practical decision-making process, IFS and PFS can be treated as two special forms of the q-ROFS for $q=1$ and $q=2$, respectively. The $\mathrm{q}$-ROFSs have gradually drawn attention of scholars in recent years and many excellent research results have been achieved, for example, the q-rung orthopair fuzzy approximate reasoning [7], integrals and differentials of q-rung orthopair fuzzy functions [8-10], various q-rung orthopair aggregation operators [11-15], q-rung orthopair fuzzy information measures [16-18], and lots of different q-rung orthopair fuzzy decision-making methods $[19,20]$.

To quantify the satisfying degrees and nonsatisfying degrees in terms of interval values instead of a single numeric value, IVq-ROFS [21, 22] has recently been developed. Jin [23] developed an interval-valued q-rung orthopair fuzzy-additive ratio assessment method. Yang et al. [24] investigated fusion strategies of continuous 
interval-valued q-rung orthopair fuzzy information, Yang and Chang [25] proposed the interval q-rung orthopair fuzzy power Muirhead mean operator. As an extension of interval-valued PFS from the perspective of the constraint condition, IVq-ROFSs have wide application fields, especially in decision-making fields. In order to well handle complex MADM or multiattribute group decision-making (MAGDM) problems under IVq-ROFSs environment, it is necessary to develop correspondingly effective decision-making methods. QUALIFLEX (qualitative flexible multiple criteria method) [26] is one of the well-known outranking methods to solve the MADM problems with exact real numbers. The most important characteristic of the QUALIFLEX method is the correct treatment of cardinal and ordinal information [4]. To adapt different fuzzy decision-making environments, several excellent studies have been devoted to fuzzy extensions of the QUALIFLEX approach in the literature. For example, Chen et al. $[27,28]$ proposed interval type-2 trapezoidal fuzzy QUALIFLEX method, Zhang and Xu [29] developed hesitant fuzzy QUALIFLEX method, and Chen [30] developed an interval-valued intuitionistic fuzzy QUALIFLEX method. More recently, some new extensions, Pythagorean fuzzy QUALIFLEX method [4], heterogeneous QUALIFLEX method [31], and SMAA-QUALIFLEX methodology with q-rung fuzzy sets [20], have been developed. Although these aforementioned methods are very effective for solving different issues, they fail to deal with IVq-ROFSs decision data in the complex decision process.

Therefore, this paper attempts to develop a new intervalvalued q-rung orthopair fuzzy QUALIFLEX decision analysis method with Dombi operators. Dombi operations developed by Domb [32] have an advantage of flexibility within the working behavior of parameters [33] and have been extended into various fuzzy environments [33-35]. Thus, this paper first defines the interval-valued q-rung orthopair fuzzy Dombi operations based on the $\mathrm{t}$-module and $\mathrm{t}$-comodule, including interval-valued q-rung orthopair fuzzy Dombi addition, multiplication, number multiplication, and power operations and investigates the relevant operation properties. Secondly, using the developed interval-valued q-rung orthopair fuzzy Dombi operations, the interval-valued q-rung orthopair fuzzy Dombi weighted average (IVq-ROFDWA) operator is proposed, and, using mathematical induction, we prove that the calculated results are still interval-valued q-rung orthopair fuzzy numbers (IVq-ROFNs), and at the same time we prove that the IVq-ROFDWA operator satisfies the properties of idempotency, commutativity, boundedness, and monotonicity. Thirdly, with the help of IVq-ROFDWA operator, we propose a new weight-determining method for identifying the weights of experts. Fourthly, the concordance/discordance index and the weighted concordance/discordance indices under IVq-ROFNs environment can be calculated and the ranking order of alternatives can be determined by comparing the comprehensive index. Finally, the algorithm of the developed interval-valued q-rung orthopair fuzzy QUALIFLEX decision analysis method is introduced.

The remainder of this paper is constructed as follows: Section 2 recalls the knowledge of IVq-ROFSs and proposes the new distance measure for IVq-ROFSs. Section 3 develops the interval-valued q-rung orthopair fuzzy Dombi operations and the IVq-ROFDWA operator. Section 4 proposes an interval-valued q-rung orthopair fuzzy QUALIFLEX decision analysis method. An illustrating example is presented in Section 5 to demonstrate the feasibility and superiority of the proposed method. Section 6 summarizes this study.

\section{Basic Concepts and New Distance Measure}

This section introduces the definition and operation of IVqROFS involved in this paper and proposes the new distance measure for IVq-ROFNs.

Definition 1 (see [5]). Let $X$ be a fixed set; a q-rung orthopair fuzzy set (q-ROFS) $A$ in $X$ is an object of the form $A=$ $\left\{<x, u_{A}(x), v_{A}(x)>\mid x \in X\right\} . \mu_{A}(x)$ and $v_{A}(x)$ are the degree of membership and the degree of nonmembership of the element $x \in X$ to $A$, respectively, and they satisfy

$$
0 \leq\left(u_{A}(x)\right)^{q}+\left(v_{A}(x)\right)^{q} \leq 1 .
$$

The degree of hesitation $\pi_{A}(x)$ can be expressed as follows:

$$
\pi_{A}(x)=\sqrt[q]{1-\left(u_{A}(x)\right)^{q}+\left(v_{A}(x)\right)^{q}}, \quad(q \geq 1) .
$$

Definition 2 (see $[21,22]$ ). Let $X$ be an ordinary finite nonempty set; the IVq-ROFS $A$ in $X$ is defined as

$$
A=\left\{<x, u_{A}(x), v_{A}(x)>\mid x \in X\right\} .
$$

The membership function is a mapping range of values to meet $u_{A}(x)=\left[u_{A}^{-}(x), u_{A}^{+}(x)\right] \subseteq[0,1]$ and the nonmembership function is a mapping range of values to meet $v_{A}(x)=\left[v_{A}^{-}(x), v_{A}^{+}(x)\right] \subseteq[0,1] ; 0 \leq\left(u_{A}^{+}(x)\right)^{q}+\left(v_{A}^{+}(x)\right)^{q} \leq$ $1,(q \geq 1)$.

The degree of hesitation of $A$ is shown in the following formula:

$$
\begin{aligned}
\pi_{A}(x) & =\left[\pi_{A}^{-}(x), \pi_{A}^{+}(x)\right] \\
& =\left[\begin{array}{c}
\sqrt[q]{1-\left(u_{A}^{+}(x)\right)^{q}-\left(v_{A}^{+}(x)\right)^{q}}, \\
\sqrt[q]{1-\left(u_{A}^{-}(x)\right)^{q}-\left(v_{A}^{-}(x)\right)^{q}}
\end{array}\right], \quad(q \geq 1 .)
\end{aligned}
$$

Definition 3 (see $[21,22])$. Let $a=\left(\left[u^{-}, u^{+}\right],\left[v^{-}, v^{+}\right]\right)$, $a_{1}=\left(\left[u_{a_{1}}^{-}, u_{a_{1}}^{+}\right],\left[v_{a_{1}}^{-}, v_{a_{1}}^{+}\right]\right)$, and $a_{2}=\left(\left[u_{a_{2}}^{-}, u_{a_{2}}^{+}\right],\left[v_{a_{2}}^{-}, v_{a_{2}}^{+}\right]\right)$be three IVq-ROFNs and $\lambda>0$; the basic operations on them are given as follows: 


$$
\begin{aligned}
a_{1} \oplus a_{2} & =\left(\left[\sqrt[q]{\left(u_{a_{1}}^{-}\right)^{q}+\left(u_{a_{2}}^{-}\right)^{q}-\left(u_{a_{1}}^{-}\right)^{q}\left(u_{a_{2}}^{-}\right)^{q}}, \sqrt[q]{\left(u_{a_{1}}^{+}\right)^{q}+\left(u_{a_{2}}^{+}\right)^{q}-\left(u_{a_{1}}^{+}\right)^{q}\left(u_{a_{2}}^{+}\right)^{q}}\right],\left[v_{a_{1}}^{-} v_{a_{2}}^{-}, v_{a_{1}}^{+} v_{a_{2}}^{+}\right]\right) \\
a_{1} \otimes a_{2} & =\left(\left[u_{a_{1}}^{-} u_{a_{2}}^{-}, u_{a_{1}}^{+} u_{a_{2}}^{+}\right],\left[\sqrt[q]{\left(v_{a_{1}}^{-}\right)^{q}+\left(v_{a_{2}}^{-}\right)^{q}-\left(v_{a_{1}}^{-}\right)^{q}\left(v_{a_{2}}^{-}\right)^{q}}, \sqrt[q]{\left(v_{a_{1}}^{+}\right)^{q}+\left(v_{a_{2}}^{+}\right)^{q}-\left(v_{a_{1}}^{+}\right)^{q}\left(v_{a_{2}}^{+}\right)^{q}}\right]\right) \\
\lambda a & =\left(\left[\sqrt[q]{1-\left(1-\left(u^{-}\right)^{q}\right)^{\lambda}}, \sqrt[q]{1-\left(1-\left(u^{+}\right)^{q}\right)^{\lambda}}\right],\left[\left(v^{-}\right)^{\lambda},\left(v^{+}\right)^{\lambda}\right]\right) \\
a^{\lambda} & =\left(\left[\left(u^{-}\right)^{\lambda},\left(u^{+}\right)^{\lambda}\right],\left[\sqrt[q]{1-\left(1-\left(v^{-}\right)^{q}\right)^{\lambda}}, \sqrt[q]{1-\left(1-\left(v^{+}\right)^{q}\right)^{\lambda}}\right]\right) .
\end{aligned}
$$

Definition 4 (see [21, 22]). Given an IVq-ROFN $a=\left(\left[u^{-}, u^{+}\right],\left[v^{-}, v^{+}\right]\right)$, the scoring function of $a$ is shown in the following formula:

$$
S(a)=\frac{1}{2}\left[\left(u_{a}^{-}\right)^{q}+\left(u_{a}^{+}\right)^{q}-\left(v_{a}^{-}\right)^{q}-\left(v_{a}^{+}\right)^{q}\right], \quad(q \geq 1) .
$$

Definition 5 (see [21, 22]). Given an IVq-ROFN $a=\left(\left[u_{a}^{-}, u_{a}^{+}\right],\left[v_{a}^{-}, v_{a}^{+}\right]\right)$, its accuracy value function is defined as follows:

$$
H(a)=\frac{1}{2}\left[\left(u_{a}^{-}\right)^{q}+\left(u_{a}^{+}\right)^{q}+\left(v_{a}^{-}\right)^{q}+\left(v_{a}^{+}\right)^{q}\right], \quad(q \geq 1) .
$$

Definition 6 (see $[21,22])$. Given two IVq-ROFNs $a_{1}=$ $\left(\left[u_{a_{1}}^{-}, u_{a_{1}}^{+}\right],\left[v_{a_{1}}^{-}, v_{a_{1}}^{+}\right]\right)$and $a_{2}=\left(\left[u_{a_{2}}^{-}, u_{a_{2}}^{+}\right],\left[v_{a_{2}}^{-}, v_{a_{2}}^{+}\right]\right)$, the comparison law of IVq-ROFNs is proposed as follows:

(1) If $S\left(a_{1}\right)>S\left(a_{2}\right)$, then $a_{1}>a_{2}$;

(2) If $S\left(a_{1}\right)<S\left(a_{2}\right)$, then $a_{1}<a_{2}$;

(3) If $S\left(a_{1}\right)=S\left(a_{2}\right)$ and

(a) if $H\left(a_{1}\right)>H\left(a_{2}\right)$, then $a_{1}>a_{2}$;

(b) if $H\left(a_{1}\right)<H\left(a_{2}\right)$, then $a_{1}<a_{2}$;

(c) if $H\left(a_{1}\right)=H\left(a_{2}\right)$, then $a_{1}=a_{2}$.
Definition 7. Given two IVq-ROFNs $a_{1}=\left(\left[u_{a_{1}}^{-}, u_{a_{1}}^{+}\right],\left[v_{a_{1}}^{-}\right.\right.$, $\left.\left.v_{a_{1}}^{+}\right]\right)$and $a_{2}=\left(\left[u_{a_{2}}^{-}, u_{a_{2}}^{+}\right],\left[v_{a_{2}}^{-}, v_{a_{2}}^{+}\right]\right)$and $q \geq 1$, the distance measure between them is developed as follows:

$$
\begin{aligned}
d\left(a_{1}, a_{2}\right)= & \frac{1}{4}\left(\left|\left(u_{a_{1}}^{-}\right)^{q}-\left(u_{a_{2}}^{-}\right)^{q}\right|+\left|\left(u_{a_{1}}^{+}\right)^{q}-\left(u_{a_{2}}^{+}\right)^{q}\right|\right. \\
& +\left|\left(v_{a_{1}}^{-}\right)^{q}-\left(v_{a_{2}}^{-}\right)^{q}\right|+\left|\left(v_{a_{1}}^{+}\right)^{q}-\left(v_{a_{2}}^{+}\right)^{q}\right| \\
& \left.+\left|\left(\pi_{a_{1}}^{-}\right)^{q}-\left(\pi_{a_{2}}^{-}\right)^{q}\right|+\left|\left(\pi_{a_{1}}^{+}\right)^{q}-\left(\pi_{a_{2}}^{+}\right)^{q}\right|\right) .
\end{aligned}
$$

Theorem 1. Let $a, b, c$ be three IVq-ROFNs; then the following results hold:

(1) $0 \leq d(a, b) \leq 1, d(a, b)=0$, if and only if $a=b$;

(2) $d(a, b)=d(b, a)$;

(3) $d(a, b) \leq d(a, c)+d(c, b)$.

Proof. It is easy to see that (2) holds according to the definition of distance measure; we next prove (1) and (3) as follows:

(1) $\quad d(a, b)=\frac{1}{4}\left(\left|\left(u_{a}^{-}\right)^{q}-\left(u_{b}^{-}\right)^{q}\right|+\left|\left(u_{a}^{+}\right)^{q}-\left(u_{b}^{+}\right)^{q}\right|+\left|\left(v_{a}^{-}\right)^{q}-\left(v_{b}^{-}\right)^{q}\right|+\left|\left(v_{a}^{+}\right)^{q}-\left(v_{b}^{+}\right)^{q}\right|+\left|\left(\pi_{a}^{-}\right)^{q}-\left(\pi_{b}^{-}\right)^{q}\right|\right.$

$$
\begin{aligned}
& \left.+\left|\left(\pi_{a}^{+}\right)^{q}-\left(\pi_{b}^{+}\right)^{q}\right|\right) \leq \frac{1}{4}\left(\left(u_{a}^{-}\right)^{q}+\left(v_{a}^{-}\right)^{q}\right. \\
& \left.+\left(\pi_{a}^{-}\right)^{q}\right)+\left(\left(u_{b}^{-}\right)^{q}+\left(v_{b}^{-}\right)^{q}+\left(\pi_{b}^{-}\right)^{q}\right)+\left(\left(u_{a}^{+}\right)^{q}+\left(v_{a}^{+}\right)^{q}+\left(\pi_{a}^{+}\right)^{q}\right)+\left(\left(u_{b}^{+}\right)^{q}\right. \\
& \left.+\left(v_{b}^{+}\right)^{q}+\left(\pi_{b}^{+}\right)^{q}\right) \leq \frac{1}{4}(1+1+1+1) \leq 1 .
\end{aligned}
$$


According to Definition 7 , it is easy to obtain $0 \leq d(a, b)$ and $d(a, b)=0$ if and only if $a=b$. Obviously, (1) holds.
According to Definition 7, it is easy to obtain

$$
\begin{aligned}
d(a, b)= & \frac{1}{4}\left(\left|\left(u_{a}^{-}\right)^{q}-\left(u_{c}^{-}\right)^{q}+\left(u_{c}^{-}\right)^{q}-\left(u_{b}^{-}\right)^{q}\right|+\left|\left(u_{a}^{+}\right)^{q}-\left(u_{c}^{+}\right)^{q}+\left(u_{c}^{+}\right)^{q}-\left(u_{b}^{+}\right)^{q}\right|+\left|\left(v_{a}^{-}\right)^{q}-\left(v_{c}^{-}\right)^{q}+\left(v_{c}^{-}\right)^{q}-\left(v_{b}^{-}\right)^{q}\right|\right. \\
& \left.+\left|\left(v_{a}^{+}\right)^{q}-\left(v_{c}^{+}\right)^{q}+\left(v_{c}^{+}\right)^{q}-\left(v_{b}^{+}\right)^{q}\right|+\left|\left(\pi_{a}^{-}\right)^{q}-\left(\pi_{c}^{-}\right)^{q}+\left(\pi_{c}^{-}\right)^{q}-\left(\pi_{b}^{-}\right)^{q}\right|+\left|\left(\pi_{a}^{+}\right)^{q}-\left(\pi_{c}^{+}\right)^{q}+\left(\pi_{a c}^{+}\right)^{q}-\left(\pi_{b}^{+}\right)^{q}\right|\right) \\
\leq & \frac{1}{4}\left(\left|\left(u_{a}^{-}\right)^{q}-\left(u_{c}^{-}\right)^{q}\right|+\left|\left(u_{c}^{-}\right)^{q}-\left(u_{b}^{-}\right)^{q}\right|+\left|\left(u_{a}^{+}\right)^{q}-\left(u_{c}^{+}\right)^{q}\right|+\left|\left(u_{c}^{+}\right)^{q}-\left(u_{b}^{+}\right)^{q}\right|+\left|\left(v_{a}^{-}\right)^{q}-\left(v_{c}^{-}\right)^{q}\right|+\left|\left(v_{c}^{-}\right)^{q}-\left(v_{b}^{-}\right)^{q}\right|+\left|\left(v_{a}^{+}\right)^{q}-\left(v_{c}^{+}\right)^{q}\right|\right. \\
& \left.+\left|\left(v_{c}^{+}\right)^{q}-\left(v_{b}^{+}\right)^{q}\right|+\left|\left(\pi_{a}^{-}\right)^{q}-\left(\pi_{c}^{-}\right)^{q}\right|+\left|\left(\pi_{c}^{-}\right)^{q}-\left(\pi_{b}^{-}\right)^{q}\right|+\left|\left(\pi_{a}^{+}\right)^{q}-\left(\pi_{c}^{+}\right)^{q}\right|+\left|\left(\pi_{a c}^{+}\right)^{q}-\left(\pi_{b}^{+}\right)^{q}\right|\right) \leq d(a, c)+d(c, b) .
\end{aligned}
$$

Obviously, (3) holds.

\section{Interval-Valued q-Rung Orthopair Fuzzy Dombi Operator}

\subsection{Interval-Valued q-Rung Orthopair Fuzzy Dombi Operation}

Definition 8 (see [32]). For any two real numbers $a, b \in[0,1]$, the Dombi sum and Dombi product of $a$ and $b$ are defined as follows:

$$
\begin{aligned}
& a \oplus_{D} b=\frac{1}{1+\left\{(a /(1-a))^{\varphi}+(b /(1-b))^{\varphi}\right\}^{(1 / \varphi)}}, \\
& a \otimes_{D} b=\frac{1}{1+\left\{((1-a) / a)^{\varphi}+((1-b) / b)^{\varphi}\right\}^{1 / \varphi}},
\end{aligned}
$$

$a_{1} \oplus_{D} a_{2}=\left(\begin{array}{l}{\left[\sqrt{1-\frac{1}{1+\left\{\left(\left(u_{a_{1}}^{-}\right)^{q} /\left(1-\left(u_{a_{1}}^{-}\right)^{q}\right)\right)^{\varphi}+\left(\left(u_{a_{2}}^{-}\right)^{q} /\left(1-\left(u_{a_{2}}^{-}\right)^{q}\right)\right)^{\varphi}\right\}^{1 / \varphi}}}, \sqrt[p]{\left.1-\frac{1}{1+\left\{\left(\left(u_{a_{1}}^{+}\right)^{q} /\left(1-\left(u_{a_{1}}^{+}\right)^{q}\right)\right)^{\varphi}+\left(\left(u_{a_{2}}^{+}\right)^{q} /\left(1-\left(u_{a_{2}}^{+}\right)^{q}\right)\right)^{\varphi}\right\}^{1 / \varphi}}\right],}\right.} \\ {\left[\sqrt[p]{\frac{1}{1+\left\{\left(\left(1-\left(v_{a_{1}}^{-}\right)^{q}\right) /\left(v_{a_{1}}\right)^{q}\right)^{\varphi}+\left(\left(1-\left(v_{a_{2}}^{-}\right)^{q}\right) /\left(v_{a_{2}}^{-}\right)^{q}\right)^{\varphi}\right\}^{1 / \varphi}}}, \sqrt[p]{\left.\frac{1}{1+\left\{\left(\left(1-\left(v_{a_{1}}^{+}\right)^{q}\right) /\left(v_{a_{1}}^{+}\right)^{q}\right)^{\varphi}+\left(\left(1-\left(v_{a_{2}}^{+}\right)^{q}\right) /\left(v_{a_{2}}^{+}\right)^{q}\right)^{\varphi}\right\}^{1 / \varphi}}\right]}\right],}\end{array}\right.$

$$
a_{1} \otimes_{D} a_{2}=\left(\left[\begin{array}{l}
{\left[\sqrt[p]{\frac{1}{1+\left\{\left(\left(u_{a_{1}}^{-}\right)^{q} /\left(1-\left(u_{a_{1}}^{-}\right)^{q}\right)\right)^{\varphi}+\left(\left(u_{a_{2}}^{-}\right)^{q} /\left(1-\left(u_{a_{2}}^{-}\right)^{q}\right)\right)^{\varphi}\right\}^{1 / \varphi}}}, \sqrt[p]{\left.\frac{1}{1+\left\{\left(\left(u_{a_{1}}^{+}\right)^{q} /\left(1-\left(u_{a_{1}}^{+}\right)^{q}\right)\right)^{\varphi}+\left(\left(u_{a_{2}}^{+}\right)^{q} /\left(1-\left(u_{a_{2}}^{+}\right)^{q}\right)\right)^{\varphi}\right\}^{1 / \varphi}}\right],}\right.} \\
{\left[\sqrt{1-\frac{1}{\left.1+\left\{\left(\left(1-\left(v_{a_{1}}^{-}\right)^{q}\right) /\left(v_{a_{1}}^{-}\right)^{q}\right)^{\varphi}+\left(\left(1-v_{a_{2}}^{-}\right)^{q}\right) /\left(v_{a_{2}}^{-}\right)^{q}\right)^{\varphi}\right\}^{1 / \varphi}}}, \sqrt{\left.1-\frac{1}{1+\left\{\left(\left(1-\left(v_{a_{1}}^{+}\right)^{q}\right) /\left(v_{a_{1}}^{+}\right)^{q}\right)^{\varphi}+\left(\left(1-\left(v_{a_{2}}^{+}\right)^{q}\right) /\left(v_{a_{2}}^{+}\right)^{q}\right)^{\varphi}\right\}^{1 / \varphi}}\right]}\right],}
\end{array}\right]\right.
$$




$$
\begin{gathered}
\left.\delta a=\left(\begin{array}{c}
{\left[\sqrt[q]{1-\frac{1}{1+\left\{\delta\left(\left(u_{a}^{-}\right)^{q} /\left(1-\left(u_{a}^{-}\right)^{q}\right)\right)^{\varphi}\right\}^{1 / \varphi}}}, \sqrt[q]{1-\frac{1}{1+\left\{\delta\left(\left(u_{a}^{+}\right)^{q} /\left(1-\left(u_{a}^{+}\right)^{q}\right)\right)^{\varphi}\right\}^{1 / \varphi}}}\right],} \\
a^{\delta}=\left(\left[\sqrt[q]{\frac{1}{1+\left\{\delta\left(\left(1-\left(v_{a}^{-}\right)^{q}\right) /\left(v_{a}^{-}\right)^{q}\right)^{\varphi}\right\}^{1 / \varphi}}}, \sqrt[q]{\left.\frac{1}{1+\left\{\delta\left(\left(1-\left(v_{a}^{+}\right)^{q}\right) /\left(u_{a}^{+}\right)^{q}\right)^{\varphi}\right\}^{1 / \varphi}}\right]}\right),\right. \\
{\left[\sqrt[q]{\frac{1+\left\{\delta\left(\left(u_{a}^{-}\right)^{q} /\left(1-\left(u_{a}^{-}\right)^{q}\right)\right)^{\varphi}\right\}^{1 / \varphi}}{1+\left\{\delta\left(\left(u_{a}^{+}\right)^{q} /\left(1-\left(u_{a}^{+}\right)^{q}\right)\right)^{\varphi}\right\}^{1 / \varphi}}}\right],\left[\sqrt[q]{1-\frac{1}{1+\left\{\delta\left(\left(v_{a}^{-}\right)^{q} /\left(1-\left(v_{a}^{-}\right)^{q}\right)\right)^{\varphi}\right\}^{1 / \varphi}}}\right]}
\end{array}\right]\right) .
\end{gathered}
$$

It can be verified that interval-valued q-rung orthopair fuzzy Dombi addition operation and multiplication operation given in this paper satisfy the operation rules of t-conorms and t-norms. Number multiplication and power Dombi operations of IVq-ROFNs also satisfy the operation rules of $\mathrm{t}$-norms and $\mathrm{t}$-conorms.

Theorem 2. Let $a=\left(\left[u_{a}^{-}, u_{a}^{+}\right],\left[v_{a}^{-}, v_{a}^{+}\right]\right), a_{1}=\left(\left[u_{a_{1}}^{-}, u_{a_{1}}^{+}\right]\right.$, $\left.\left[v_{a_{1}}^{-}, v_{a_{1}}^{+}\right]\right)$, and $a_{2}=\left(\left[u_{a_{2}}^{-}, u_{a_{2}}^{+}\right],\left[v_{a_{2}}^{-}, v_{a_{2}}^{+}\right]\right)$be three IVqROFNs, and $\delta, \delta_{1}, \delta_{2}>0$; then the six following properties are satisfied and are shown in the six following equations:

$$
\begin{aligned}
& a_{1} \oplus_{D} a_{2}=a_{2} \oplus_{D} a_{1}, \\
& a_{1} \otimes_{D} a_{2}=a_{2} \otimes_{D} a_{1},
\end{aligned}
$$

$$
\begin{aligned}
\delta\left(a_{1} \oplus_{D} a_{2}\right) & =\left(\delta a_{2}\right) \oplus_{D}\left(\delta a_{1}\right) \\
\left(a_{1} \otimes_{D} a_{2}\right)^{\delta} & =\left(a_{2}\right)^{\delta} \otimes_{D}\left(a_{1}\right)^{\delta} \\
\delta_{1} a \oplus_{D} \delta_{2} a & =\left(\delta_{1}+\delta_{2}\right) a \\
(a)^{\delta_{1}} \otimes_{D}(a)^{\delta_{2}} & =a^{\left(\delta_{1}+\delta_{2}\right)}
\end{aligned}
$$

Theorem 2 can be proved by the interval-valued q-rung orthopair fuzzy Dombi operation laws. We only prove (18) and the others can be proved similarly.

Proof. According to (12) and (14), the value of $\delta\left(a_{1} \oplus_{D} a_{2}\right)$ can be obtained as

$\delta\left(a_{1} \oplus_{D} a_{2}\right)=\left(\begin{array}{l}{\left[\sqrt[p]{1-\frac{1}{1+\left\{\delta\left(\left(\left(u_{a_{1}}^{-}\right)^{q} /\left(1-\left(u_{a_{1}}^{-}\right)^{q}\right)\right)^{\varphi}+\left(\left(u_{a_{2}}^{-}\right)^{q} /\left(1-\left(u_{a_{2}}^{-}\right)^{q}\right)\right)^{\varphi}\right)\right\}^{1 / \varphi}}}, \sqrt{\left.1-\frac{1}{1+\left\{\delta\left(\left(\left(u_{a_{1}}^{+}\right)^{q} /\left(1-\left(u_{a_{1}}^{+}\right)^{q}\right)\right)^{\varphi}+\left(\left(u_{a_{2}}^{+}\right)^{q} /\left(1-\left(u_{a_{2}}^{+}\right)^{q}\right)\right)^{\varphi}\right)\right\}^{1 / \varphi}}\right],}\right],} \\ {\left[\sqrt{\frac{1}{\left.1+\left\{\delta\left(\left(\left(1-\left(v_{a_{1}}^{-}\right)^{q}\right) /\left(v_{a_{1}}^{-}\right)^{q}\right)^{\varphi}+\left(\left(1-v_{a_{2}}^{-}\right)^{q}\right) /\left(v_{a_{2}}^{-}\right)^{q}\right)^{\varphi}\right)\right\}^{1 / \varphi}}}, \sqrt[p]{\left.\frac{1}{\left.1+\left\{\delta\left(\left(\left(1-\left(v_{a_{1}}^{+}\right)^{q}\right) /\left(v_{a_{1}}^{+}\right)^{q}\right)^{\varphi}+\left(\left(1-v_{a_{2}}^{+}\right)^{q}\right) /\left(v_{a_{2}}^{+}\right)^{q}\right)^{\varphi}\right)\right\}^{1 / \varphi}}\right]}\right]}\end{array}\right.$

and, according to (14), $\delta a_{1}$ and $\delta a_{2}$ are

$$
\delta a_{1}=\left(\begin{array}{l}
{\left[\sqrt{\left[1-\frac{1}{1+\left\{\delta\left(\left(u_{a_{1}}^{-}\right)^{q} /\left(1-\left(u_{a_{1}}^{-}\right)^{q}\right)\right)^{\varphi}\right\}^{1 / \varphi}},\right.}, \sqrt[1]{\left.1-\frac{1}{1+\left\{\delta\left(\left(u_{a_{1}}^{+}\right)^{q} /\left(1-\left(u_{a_{1}}^{+}\right)^{q}\right)\right)^{\varphi}\right\}^{1 / \varphi}}\right]}\right]} \\
{\left[\sqrt[p]{\frac{1}{1+\left\{\delta\left(1-\left(v_{a_{1}}^{-}\right)^{q} /\left(\left(v_{a_{1}}^{-}\right)^{q}\right)\right)^{\varphi}\right\}^{1 / \varphi}}}, \sqrt[1]{\frac{1+\left\{\delta\left(\left(1-\left(v_{a_{1}}^{+}\right)^{q}\right) /\left(v_{a_{1}}^{+}\right)^{q}\right)^{\varphi}\right\}^{1 / \varphi}}{1+\{}}\right]}
\end{array}\right),
$$




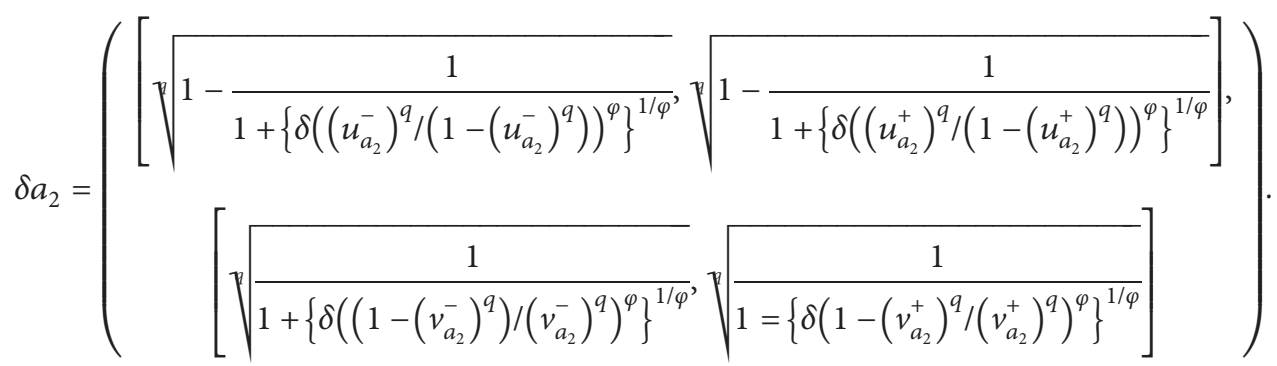

According to (12), $\left(\delta a_{2}\right) \oplus_{D}\left(\delta a_{1}\right)$ can be obtained as

$\left(\delta a_{1}\right) \oplus_{D}\left(\delta a_{2}\right)=\left(\begin{array}{l}{\left[\sqrt{1-\frac{1}{1+\left\{\delta\left(\left(u_{a_{1}}^{-}\right)^{q} /\left(1-\left(u_{a_{1}}^{-}\right)^{q}\right)\right)^{\varphi}+\left(\left(u_{a_{2}}^{-}\right)^{q} /\left(1-\left(u_{a_{2}}^{-}\right)^{q}\right)\right)^{\varphi}\right\}^{1 / \varphi}},}, \sqrt[1]{\left.1-\frac{1}{1+\left\{\delta\left(\left(u_{a_{1}}^{+}\right)^{q} /\left(1-\left(u_{a_{1}}^{+}\right)^{q}\right)\right)^{\varphi}+\left(\left(u_{a_{2}}^{+}\right)^{q} /\left(1-\left(u_{a_{2}}^{+}\right)^{q}\right)\right)^{\varphi}\right\}^{1 / \varphi}}\right],}\right]} \\ {\left[\sqrt{\frac{1}{1+\left\{\delta\left(\left(1-\left(v_{a_{1}}^{-}\right)^{q}\right) /\left(v_{a_{1}}^{-}\right)^{q}\right)^{\varphi}+\left(\left(1-\left(v_{a_{2}}^{-}\right)^{q} /\left(v_{a_{2}}^{-}\right)^{q}\right)^{\varphi}\right\}^{1 / \varphi}\right.}}, \sqrt[p]{\left.\frac{1}{1+\left\{\delta\left(\left(1-\left(v_{a_{1}}^{+}\right)^{q}\right) /\left(v_{a_{1}}^{+}\right)^{q}\right)^{\varphi}+\left(\left(1-\left(v_{a_{2}}^{+}\right)^{q}\right) /\left(v_{a_{2}}^{+}\right)^{q}\right)^{\varphi}\right\}^{1 / \varphi}}\right]}\right]}\end{array}\right.$

Therefore, $\delta\left(a_{1} \oplus_{D} a_{2}\right)=\left(\delta a_{2}\right) \oplus_{D}\left(\delta a_{1}\right)$ in (18) is certified.

\subsection{Interval-Valued q-Rung Orthopair Fuzzy Dombi} Weighted Average Operator

Definition 10. Let $a_{j}=\left(\left[u_{a_{j}}^{-}, u_{a_{j}}^{+}\right],\left[v_{a_{j}}^{-}, v_{a_{j}}^{+}\right]\right)(j=1,2, \ldots, n)$ be a set of IVq-ROFNs; $\omega=\left(\omega_{1}, \omega_{2}, \ldots, \omega_{n}\right)^{T}$ for the weight vector and satisfies $\sum_{j=1}^{n} \omega_{j}=1, \omega_{j} \in[0,1],(j=1,2, \ldots, n)$. Then, the IVqROFDWA operator is defined as follows:

$$
\operatorname{IVq}-\operatorname{ROFDWA}\left(a_{1}, a_{2}, \ldots, a_{n}\right)=\oplus_{D i=1}^{n} \omega_{i} a_{i} .
$$

Theorem 3. Let $a_{j}=\left(\left[u_{a_{i}}^{-}, u_{a_{j}}^{+}\right],\left[v_{a_{j}}^{-}, v_{a_{j}}^{+}\right]\right)(j=1,2,3, \ldots, n)$ be a set of IVq-ROFNs; the aggregation result of $\operatorname{IVq}-\operatorname{ROFDWA}\left(a_{1}, a_{2}, a_{3}, \ldots a_{n}\right)$ is shown as follows:

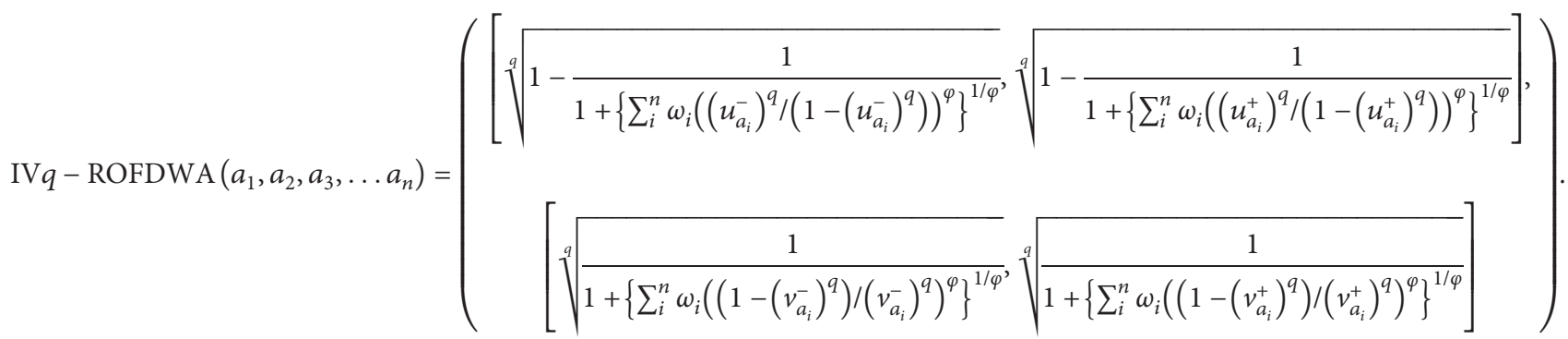


Proof.

When $n=2$,

$$
\omega_{1} a_{1}=\left(\begin{array}{l}
{\left[\sqrt[p]{1-\frac{1}{1+\left\{\omega_{1}\left(\left(u_{a_{1}}^{-}\right)^{q} /\left(1-\left(u_{a_{1}}^{-}\right)^{q}\right)\right)^{\varphi}\right\}^{1 / \varphi}},}, \sqrt[p]{\left.1-\frac{1}{1+\left\{\omega_{1}\left(\left(u_{a_{1}}^{+}\right)^{q} /\left(1-\left(u_{a_{1}}^{+}\right)^{q}\right)\right)^{\varphi}\right\}^{1 / \varphi}}\right]}\right]} \\
\omega_{2} a_{2}=
\end{array}\right],
$$

and then $\operatorname{IVq}-\operatorname{ROFDWA}\left(a_{1}, a_{2}\right)$ can be obtained as follows:

$$
\begin{aligned}
& \operatorname{IV} q-\operatorname{ROFDWA}\left(a_{1}, a_{2}\right)=\oplus_{D i=1}^{2} \omega_{i} a_{i} \\
& =\omega_{1} a_{1} \oplus_{D} \omega_{2} a_{2}
\end{aligned}
$$

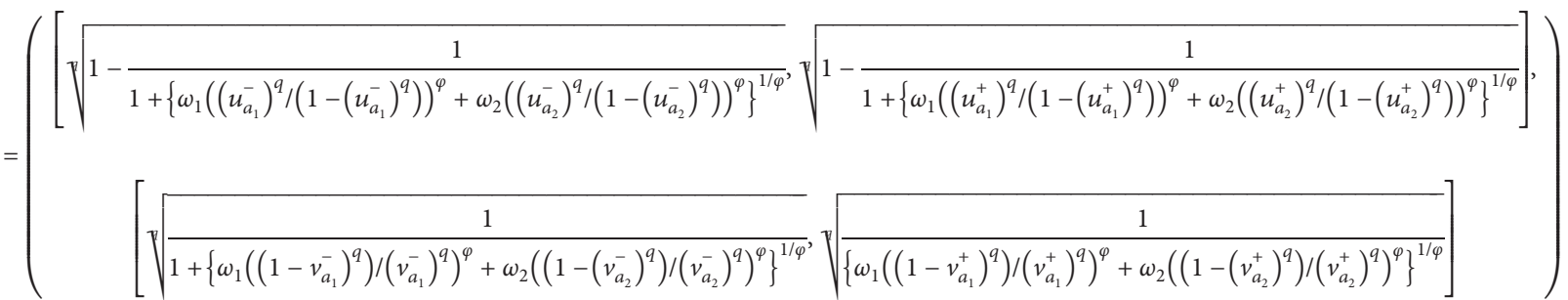

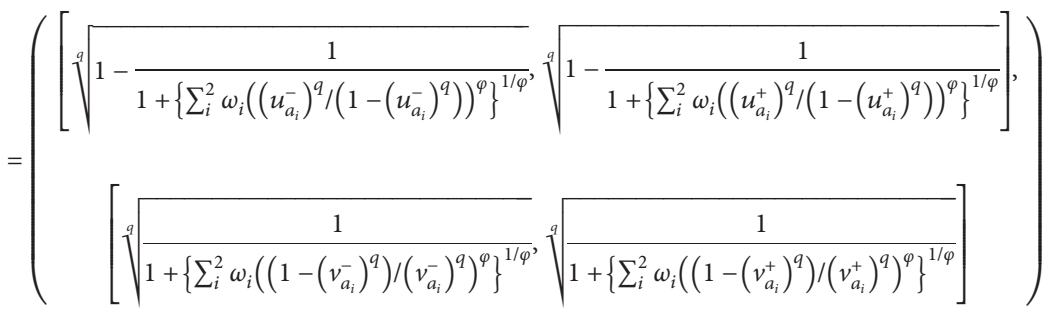

Therefore, this result holds for $n=2$. Suppose that (26) holds for $n=k$; that is to say, 
$\operatorname{IV} q-\operatorname{ROFDWA}\left(a_{1}, a_{2}, a_{3}, \ldots a_{k}\right)=\left(\begin{array}{l}{\left[\sqrt[q]{1-\frac{1}{1+\left\{\sum_{i}^{k} \omega_{i}\left(\left(u_{a_{i}}^{-}\right)^{q} /\left(1-\left(u_{a_{i}}^{-}\right)^{q}\right)\right)^{\varphi}\right\}^{1 / \varphi}}}, \sqrt[q]{1-\frac{1}{1+\left\{\sum_{i}^{k} \omega_{i}\left(\left(u_{a_{i}}^{+}\right)^{q} /\left(1-\left(u_{a_{i}}^{+}\right)^{q}\right)\right)^{\varphi}\right\}^{1 / \varphi}}}\right],} \\ {\left[\sqrt[q]{\frac{1}{1+\left\{\sum_{i}^{k} \omega_{i}\left(\left(1-\left(v_{a_{i}}^{-}\right)^{q}\right) /\left(v_{a_{i}}^{-}\right)^{q}\right)^{\varphi}\right\}^{1 / \varphi}}}, \sqrt[q]{\frac{1}{1+\left\{\sum_{i}^{k} \omega_{i}\left(\left(1-\left(v_{a_{i}}^{+}\right)^{q}\right) /\left(v_{a_{i}}^{+}\right)^{q}\right)^{\varphi}\right\}^{1 / \varphi}}}\right]}\end{array}\right]$.

When $n=k+1$, we have

$\operatorname{IV} q-\operatorname{ROFDWA}\left(a_{1}, a_{2}, a_{3}, \ldots a_{k+1}\right)=\operatorname{IV} q-\operatorname{ROFDWA}\left(a_{1}, a_{2}, a_{3}, \ldots a_{k}\right) \oplus_{D} \omega_{k+1} a_{k+1}$

$=(\begin{array}{c}{\left[\sqrt[q]{1-\frac{1}{1+\left\{\sum_{i}^{k} \omega_{i}\left(\left(u_{a_{i}}^{-}\right)^{q} /\left(1-\left(u_{a_{i}}^{-}\right)^{q}\right)\right)^{\varphi}\right\}^{1 / \varphi}},}, \sqrt[q]{\left.1-\frac{1}{1+\left\{\sum_{i}^{k} \omega_{i}\left(\left(u_{a_{i}}^{+}\right)^{q} /\left(1-\left(u_{a_{i}}^{+}\right)^{q}\right)\right)^{\varphi}\right\}^{1 / \varphi}}\right]},\right.} \\ {\left[\sqrt[q]{\frac{1}{1+\left\{\sum_{i}^{k} \omega_{i}\left(\left(1-\left(v_{a_{i}}^{-}\right)^{q}\right) /\left(v_{a_{i}}^{-}\right)^{q}\right)^{\varphi}\right\}^{1 / \varphi}}}, \sqrt[q]{\frac{1}{1+\left\{\sum_{i}^{k} \omega_{i}\left(\left(1-\left(v_{a_{i}}^{+}\right)^{q}\right) /\left(v_{a_{i}}^{+}\right)^{q}\right)^{\varphi}\right\}^{1 / \varphi}}}\right]}\end{array} \underbrace{}_{D}$

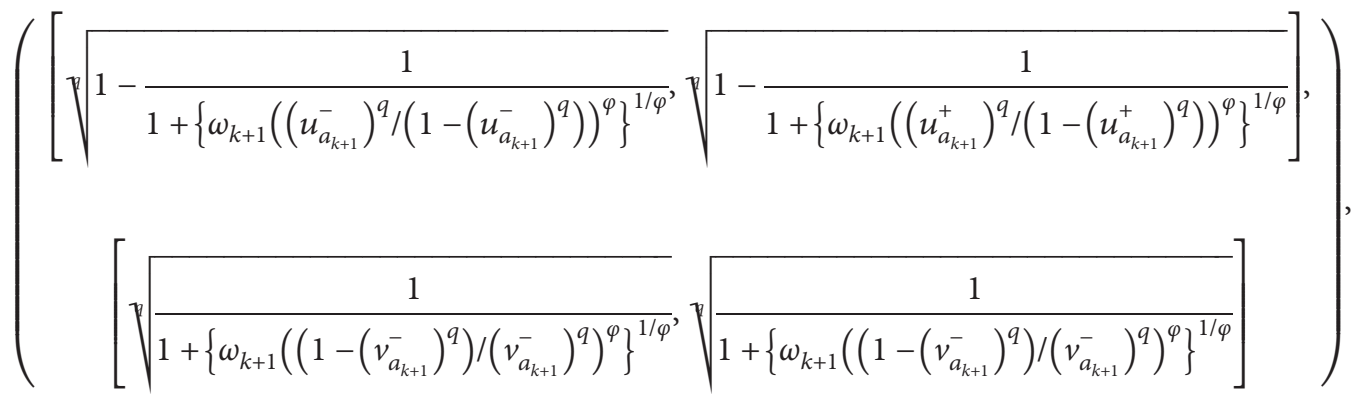

and, according to formulas (12) and (14), we get

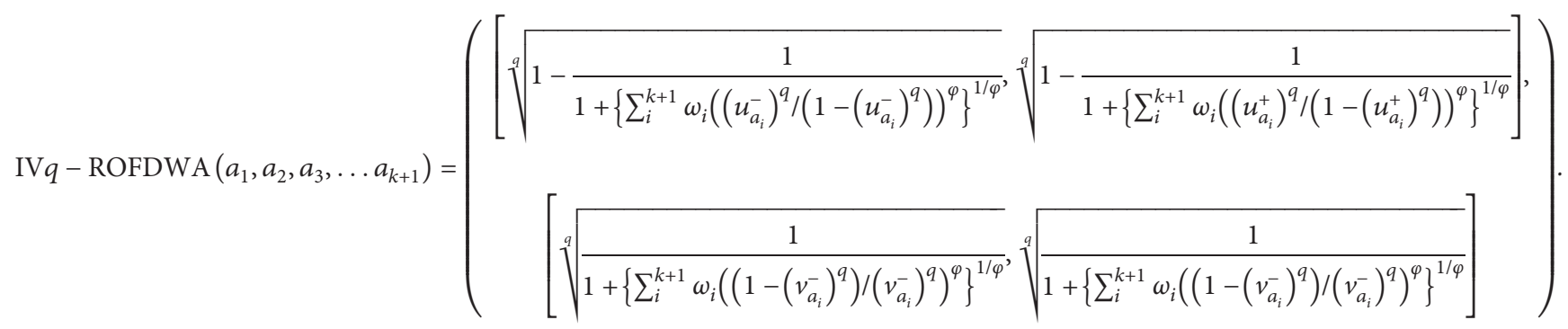


Therefore, (30) holds for $n=k+1$, and, according to the mathematical induction, Theorem 3 can be proved.

Theorem 4 (idempotency). Let $a_{j}=\left(\left[u_{a_{j}}^{-}, u_{a_{j}}^{+}\right],\left[v_{a_{j}}^{-}, v_{a_{j}}^{+}\right]\right)$ $(j=1,2,3, \ldots, n)$ be a set of IVq-ROFNs and $a_{j}=a$; then we have

$$
\operatorname{IV} q-\operatorname{ROFDWA}\left(a_{1}, a_{2}, a_{3}, \ldots, a_{n}\right)=a
$$

Proof. For any $j(j=1,2,3, \ldots, n), a_{j}=a$; then the following result can be obtained:

$$
\begin{aligned}
& \operatorname{IVq}-\operatorname{ROFDWA}\left(a_{1}, a_{2}, a_{3}, \ldots a_{n}\right)=\left(\begin{array}{c}
{\left[\sqrt[q]{1-\frac{1}{1+\left\{\sum_{i}^{n} \omega_{i}\left(\left(u_{a}^{-}\right)^{q} /\left(1-\left(u_{a}^{-}\right)^{q}\right)\right)^{\varphi}\right\}^{1 / \varphi}}}, \sqrt[q]{\left.1-\frac{1}{1+\left\{\sum_{i}^{n} \omega_{i}\left(\left(u_{a}^{+}\right)^{q} /\left(1-\left(u_{a}^{+}\right)^{q}\right)\right)^{\varphi}\right\}^{1 / \varphi}}\right],}\right.} \\
{\left[\sqrt[q]{\frac{1}{1+\left\{\sum_{i}^{n} \omega_{i}\left(\left(1-\left(v_{a}^{-}\right)^{q}\right) /\left(v_{a}^{-}\right)^{q}\right)^{\varphi}\right\}^{1 / \varphi}}}, \sqrt[q]{\frac{1}{1+\left\{\sum_{i}^{n} \omega_{i}\left(\left(1-\left(v_{a}^{+}\right)^{q}\right) /\left(v_{a}^{+}\right)^{q}\right)^{\varphi}\right\}^{1 / \varphi}}}\right]}
\end{array}\right) \\
& =\left(\begin{array}{c}
{\left[\sqrt[q]{1-\frac{1}{1+\left(\left(u_{a}^{-}\right)^{q} /\left(1-\left(u_{a}^{-}\right)^{q}\right)\right)}}, \sqrt[q]{\left.1-\frac{1}{1+\left(u_{a}^{+}\right)^{q} /\left(1-\left(u_{a}^{+}\right)^{q}\right)}\right]}\right]} \\
{\left[\sqrt[q]{\frac{1}{1+\left(1-\left(v_{a}^{-}\right)^{q}\right) /\left(v_{a}^{-}\right)^{q}}}, \sqrt[q]{\left.\frac{1}{1+\left(1-\left(v_{a}^{+}\right)^{q}\right) /\left(v_{a}^{+}\right)^{q}}\right]}\right)}
\end{array}\right) \\
& =\left(\left[u_{a}^{-}, u_{a}^{+}\right],\left[v_{a}^{-}, v_{a}^{+}\right]\right) \\
& =a
\end{aligned}
$$

Theorem 5 (boundedness). Let $a_{j}=\left(\left[u_{a_{i}}^{-}, u_{a_{j}}^{+}\right],\left[v_{a_{j}}^{-}, v_{a_{j}}^{+}\right]\right)$ $(j=1,2,3, \ldots, n)$ be a set of IVq-ROFNs; then it is easy to obtain

$$
\begin{aligned}
& a_{\min } \leq \operatorname{IVq}-\operatorname{ROFDWA}\left(a_{1}, a_{2}, a_{3}, \ldots, a_{n}\right) \leq a_{\max }, \\
& a_{\max }=\max _{j}\left(a_{j}\right), \\
& a_{\min }=\min _{j}\left(a_{j}\right) .
\end{aligned}
$$

The proof of Theorem 5 can be deduced by the intervalvalued q-rung orthopair fuzzy Dombi operations, which is ignored here.

Theorem 6 (monotonicity). Let $a_{j}, a_{j}^{*}(j=1,2, \ldots, n)$ be two sets of IVq-ROFNs, and $a_{j} \leq a_{j}^{*}(j=1,2, \ldots, n)$; then we have

$$
\begin{gathered}
\operatorname{IV} q-\operatorname{ROFDWA}\left(a_{1}, a_{2}, a_{3}, \ldots, a_{n}\right) \leq \operatorname{IV} q \\
-\operatorname{ROFDWA}\left(a_{1}^{*}, a_{2}^{*}, a_{3}^{*}, \ldots, a_{n}^{*}\right) .
\end{gathered}
$$

Proof. According to (18), we can see

$$
\begin{gathered}
I V q-\operatorname{ROF} D W A\left(a_{1}, a_{2}, a_{3}, \ldots, a_{n}\right) \\
\quad=\omega_{1} a_{1} \oplus_{D} \omega_{2} a_{2} \oplus_{D} \omega_{3} a_{3} \oplus_{D} \ldots \oplus_{D} \omega_{n} a_{n}, \\
\operatorname{IV} q-\operatorname{ROFDWA}\left(a_{1}^{*}, a_{2}^{*}, a_{3}^{*}, \ldots, a_{n}^{*}\right) \\
=\omega_{1} a_{1}^{*} \oplus_{D} \omega_{2} a_{2}^{*} \oplus_{D} \omega_{3} a_{3}^{*} \oplus_{D} \ldots \oplus_{D} \omega_{n} a_{n}^{*} .
\end{gathered}
$$

Comparing the right sides of (36) and (37), (35) holds.

Theorem 7 (commutativity). Let $\left(\alpha_{1}^{\prime}, \alpha_{2}^{\prime}, \ldots, \alpha_{n}^{\prime}\right)$ be a set of IVq-ROFNs, and let $\left(\alpha_{1}, \alpha_{2}, \ldots, \alpha_{n}\right)$ be any of the permutations of $\left(\alpha_{1}^{\prime}, \alpha_{2}^{\prime}, \ldots, \alpha_{n}^{\prime}\right)$; then, we have

$$
\begin{aligned}
& \operatorname{IV} q-\operatorname{ROFDWA}\left(\alpha_{1}, \alpha_{2}, \ldots, \alpha_{n}\right) \\
& =\operatorname{IV} q-\operatorname{ROFDWA}\left(\alpha_{1}^{\prime}, \alpha_{2}^{\prime}, \ldots, \alpha_{n}^{\prime}\right) .
\end{aligned}
$$

The proof of Theorem 7 can be deduced by the intervalvalued q-rung orthopair fuzzy Dombi operations, which is ignored here.

\section{Interval-Valued q-Rung Orthopair Fuzzy QUALIFLEX Decision Analysis Approach}

4.1. Description of MAGDM Problem with IVq-ROFNs. Let $X=\left\{x_{1}, x_{2}, \ldots, x_{m}\right\}$ be a set of alternatives, let $C=\left\{c_{1}, c_{2}, \ldots, c_{n}\right\}$ be the attributes set, and let $D=\left\{d_{1}, d_{2}, \ldots, d_{t}\right\}$ be the experts set. We denote the attribute weighting vector by $W=\left(w_{1}, w_{2}, \ldots, w_{n}\right)^{T}$, where $w_{j}$ is the relative weight of attribute $c_{j}$ and $\sum_{j=1}^{n} w_{j}=1, w_{j} \in[0,1](j=1,2, \ldots, n)$. Let $\omega_{k}$ be the weight of expert $d_{k}(k=1,2, \ldots, t)$ and the expert weights are unknown in advance in this study. In real-world MAGDM process, each expert provides an evaluated value for an alternative under one attribute, and all evaluated information provided by the expert constructs a decision matrix. For the $k$-th expert, the decision matrix is expressed as $A^{(k)}=\left(a_{i j}^{(k)}\right)_{m \times n}$, and in this study the evaluated values 
provided by the experts are represented by IVq-ROFNs and thus the element $a_{i j}^{(k)}=\left(\left[u_{a_{i j}^{(k)}}^{-}, u_{a_{i j}^{(k)}}^{+}\right],\left[v_{a_{i j}^{(k)}}^{-}, v_{a_{i j}^{(k)}}^{+}\right]\right)$in the decision matrix $A^{(k)}$ takes the form of IVq-ROFN satisfying $\left(u_{a_{i j}^{(k)}}^{+}\right)^{q}+\left(v_{a_{i j}^{(k)}}^{+}\right)^{q} \leq 1$. The value of parameter $q$ is determined by DMs according to the actual decision-making situation.

The dimensions and measurements of attribute values are often different because the types of evaluated attributes are different. To eliminate the effect of different physical dimensions and measurements on the final decision results, we transform the attribute values of the cost type into the attribute values of the benefit type by using the following equation:

$r_{i j}^{(k)}= \begin{cases}a_{i j}^{(k)}, & a_{i j}^{(k)} \in \Omega_{1}, \\ \left(a_{i j}^{(k)}\right)^{c}, & a_{i j}^{(k)} \in \Omega_{2}, \quad i=1,2, \ldots, m ; j=1,2, \ldots, n,\end{cases}$

where $\Omega_{1}$ indicates benefit type and $\Omega_{2}$ indicates cost type, and

$$
\left(a_{i j}^{(k)}\right)^{c}=\left(\left[v_{a_{i j}}^{-}, v_{a_{i j}}^{+}\right],\left[u_{a_{i j}^{-}}^{-}, u_{a_{i j}}^{+}\right]\right) .
$$

In what follows, we develop an interval-valued q-rung orthopair fuzzy QUALIFLEX analysis approach to solve the above MAGDM problems with incomplete weight information in which the evaluation values are expressed in the format of IVq-ROFNs. One important task of this method is to determine the weights of experts.

4.2. Identification of Experts' Weights. In practical group decision-making process, the group opinion should be consistent with each expert's opinion to the greatest extent $[36,37]$. Motivated by the study in [38], the group opinions in this paper are divided into four parts, that is, the positive ideal group opinion, the negative ideal group opinion, the negative ideal left group opinion, and the negative ideal right group opinion. That is to say, the weight of one expert should be assigned a greater value when the deviation value between the positive group opinion and this expert is much smaller. Similarly, the weight of one expert should be assigned a smaller value when the deviation value between the negative group opinion and this expert is much smaller.

Thus, we first denote the positive ideal group opinion by $\left(C r_{i}\right)^{+*}=\left(\left(C r_{i 1}\right)^{+^{*}},\left(C r_{i 2}\right)^{+*}, \ldots,\left(C r_{i n}\right)^{+^{*}}\right)$, and the element $\left(C r_{i j}\right)^{t^{*}}$ can be determined by the following formula:

$$
\begin{aligned}
& \left(C r_{i j}\right)^{+^{*}}=\left(\left[u_{\left(C r_{i j}\right)^{+*}}^{-} u_{\left(C r_{i j}\right)^{+*}}^{+}\right],\left[v_{\left(C r_{i j}\right)^{-*}, v_{\left(C r_{i j}\right)^{+*}}^{+}}^{+}\right]\right) \\
& =\operatorname{IV} q-\operatorname{ROFDWA}\left(r_{i j}^{(1)}, r_{i j}^{(2)}, \ldots, r_{i j}^{(t)}\right) \text {. }
\end{aligned}
$$

Then, the negative ideal group opinion is denoted by $\left(C r_{i}\right)^{-^{*}}=\left(\left(C r_{i 1}\right)^{-*},\left(C r_{i 2}\right)^{-*}, \ldots,\left(C r_{i n}\right)^{-*}\right)$, and the element $\quad\left(C r_{i j}\right)^{-^{*}}=\left(\left[u_{\left(C r_{i j}\right)^{-*}}^{-}, u_{\left(C r_{i j}\right)^{-*}}^{+}\right],\left[v_{\left(C r_{i j}\right)^{-*}}^{-}, v_{\left(C r_{i j}\right)^{-*}}^{+}\right]\right)$ can be determined according to (40) as follows:

$$
\begin{aligned}
& u_{\left(C r_{i j}\right)^{-*}}^{-}=v_{\left(C r_{i j}\right)^{+*},}^{-} \\
& u_{\left(C r_{i j}\right)^{-*}}^{+}=v_{\left(C r_{i j}\right)^{+*},} \\
& v_{\left(C r_{i j}\right)^{-*}}^{-}=u_{\left(C r_{i j}\right)^{+*},}^{-} \\
& v_{\left(C r_{i j}\right)^{+*}}^{+} u_{\left(C r_{i j}\right)^{+*}}^{+}
\end{aligned}
$$

Meanwhile, the negative ideal right opinion is denoted by $\left(I r_{i}\right)^{+^{*}}=\left(\left(I r_{i 1}\right)^{+^{*}},\left(I r_{i 2}\right)^{+^{*}}, \cdots,\left(I r_{i n}\right)^{+^{*}}\right)$ and the element $\left(I r_{i j}\right)^{+^{*}}=\left(\left[u_{\left(I r_{i j}\right)^{+^{*}}}^{-}, u_{\left(I r_{i j}\right)^{+*}}^{+}\right],\left[v_{\left(I r_{i j}\right)^{+^{*}}}^{-}, v_{\left(I r_{i j}\right)^{+*}}^{+}\right]\right)$can be determined by the following formula:

$u_{\left(I r_{i j}\right)^{+^{*}}}^{-}=\max _{k=1}^{t}\left\{u_{r_{i j}^{(k)}}^{-}\right\}, \quad u_{\left(I r_{i j}\right)^{+*}}^{+}=\max _{k=1}^{t}\left\{u_{r_{i j}^{(k)}}^{+}\right\}$,

$v_{\left(I r_{i j}\right)^{+^{*}}}^{-}=\max _{k=1}^{t}\left\{v_{r_{i j}^{(k)}}^{-}\right\}, \quad v_{\left(I r_{i j}\right)^{+*}}^{+}=\max _{k=1}^{t}\left\{v_{r_{i j}^{(k)}}^{+}\right\}$.

Similarity, the negative ideal left group opinion is denoted by $\left(I r_{i}\right)^{-^{*}}=\left(\left(I r_{i 1}\right)^{-^{*}},\left(I r_{i 2}\right)^{-^{*}}, \ldots,\left(I r_{i n}\right)^{-^{*}}\right)$ and $\left(I r_{i j}\right)^{-^{*}}=\left(\left[u_{\left(I r_{i j}\right)^{-*}}^{-}, u_{\left(I r_{i j}\right)^{-*}}^{+}\right],\left[v_{\left(I r_{i j}\right)^{-*}}^{-}, v_{\left(I r_{i j}\right)^{-*}}^{+}\right]\right)$which can be determined by the following formula:

$$
\begin{aligned}
& u_{\left(I r_{i j}\right)^{-*}}^{-}=\min _{k=1}^{t}\left\{u_{r_{i j}^{(k)}}^{-}\right\}, \\
& u_{\left(I r_{i j}\right)^{-*}}^{+}=\min _{k=1}^{t}\left\{u_{r_{i j}^{(k)}}^{+}\right\}, \\
& v_{\left(I r_{i j}\right)^{-*}}^{-}=\min _{k=1}^{t}\left\{v_{r_{i j}^{(k)}}^{-}\right\}, \\
& v_{\left(I r_{i j}\right)^{+*}}^{+}=\min _{k=1}^{t}\left\{v_{r_{i j}^{(k)}}^{+}\right\} .
\end{aligned}
$$

Then, the closeness index for expert $d_{k}$ is determined by the following formula:

$$
\begin{aligned}
& \mathrm{CI}_{k}=\frac{\sum_{j=1}^{n} \sum_{i=1}^{m} d\left(r_{i j}^{(k)},\left(C r_{i}\right)^{*^{*}}\right)+\sum_{j=1}^{n} \sum_{i=1}^{m} d\left(r_{i j}^{(k)},\left(I r_{i}\right)^{+^{*}}\right)+\sum_{j=1}^{n} \sum_{i=1}^{m} d\left(r_{i j}^{(k)},\left(I r_{i}\right)^{-^{*}}\right)}{\sum_{j=1}^{n} \sum_{i=1}^{m} d\left(r_{i j}^{(k)},\left(C r_{i}\right)^{+^{*}}\right)+\sum_{j=1}^{n} \sum_{i=1}^{m} d\left(r_{i j}^{(k)},\left(C r_{i}\right)^{-*}\right)+\sum_{j=1}^{n} \sum_{i=1}^{m} d\left(r_{i j}^{(k)},\left(I r_{i}\right)^{+^{*}}\right)+\sum_{j=1}^{n} \sum_{i=1}^{m} d\left(r_{i j}^{(k)},\left(I r_{i}\right)^{-*}\right)} \\
& \text { Thus, the weights of expert can be determined by the } \\
& \text { following formula: }
\end{aligned}
$$


4.3. Interval-Valued q-Rung Orthopair Fuzzy QUALIFLEX Method. Another important task is to sort the alternatives and identify the best optimal alternative. On the basis of the obtained weights of experts, we next integrate the individual decision matrices $R^{(k)}=\left(r_{i j}^{(k)}\right)_{m \times n}(k=1,2, \ldots, t)$ into the collective decision matrix $R=\left(r_{i j}\right)_{m \times n}$ by the developed IVq-ROFDWA operator as follows:

$$
\begin{gathered}
r_{i j}=\operatorname{IVq}-\operatorname{ROFDWA}\left(r_{i j}^{(1)}, r_{i j}^{(2)}, \ldots, r_{i j}^{(t)}\right), \\
i=1,2, \ldots, m ; j=1,2, \ldots, n .
\end{gathered}
$$

Based on the collective decision matrix, the intervalvalued q-rung orthopair fuzzy QUALIFLEX method is developed to obtain the ranking order of alternatives, which starts with the computation of the concordance/discordance index based on the successive permutations of all possible rankings of alternatives.

For the set of alternatives $X=\left\{x_{1}, x_{2}, \ldots, x_{m}\right\}$, there are $m$ ! permutations of the ranking of all alternatives, and let $P_{\rho}$ $(\rho=1,2, \ldots, m !)$ denote the $\rho^{\text {th }}$ permutation as

$$
P_{\rho}=\left(\ldots, x_{\xi}, \ldots, x_{\zeta}, \ldots\right) \text {. }
$$

$x_{\xi}, x_{\zeta} \in X$; the alternative $x_{\xi}$ is ranked higher than or equal to $x_{\zeta}$.

Considering that the decision information takes the form of IVq-ROFNs, this study utilizes the ranking approach introduced in Definition 6 to compare the magnitudes of evaluated values and compute the corresponding concordance/discordance index by the distance measure. Then, the concordance/discordance index $\phi_{j}^{\rho}\left(x_{\xi}, x_{\zeta}\right)$ for each pair of alternatives $\left(x_{\xi}, x_{\zeta}\right), x_{\xi}, x_{\zeta} \in X$, at the level of preorder according to the $j^{\text {th }}$ attribute and the ranking corresponding to the $\rho^{\text {th }}$ permutation, can be defined as follows:

$$
\phi_{j}^{\rho}\left(x_{\xi}, x_{\zeta}\right)= \begin{cases}d\left(r_{\xi j}, r_{\zeta j}\right), & \text { if } r_{\xi j}>r_{\zeta j}, \\ 0, & \text { if } r_{\xi j}=r_{\zeta j}, \\ -d\left(r_{\xi j}, r_{\zeta j}\right), & \text { if } r_{\xi j}<r_{\zeta j} .\end{cases}
$$

Thus, the weighted concordance/discordance index $\phi_{j}^{\rho}\left(x_{\xi}, x_{\zeta}\right)$ for each pair of alternatives $\left(x_{\xi}, x_{\zeta}\right)$, at the level of preorder according to $n$ attributes and the ranking corresponding to the $\rho^{\text {th }}$ permutation, can be defined as follows:

$$
\phi^{\rho}\left(x_{\xi}, x_{\zeta}\right)=\sum_{j=1}^{n}\left(w_{j} \phi_{j}^{\rho}\left(x_{\xi}, x_{\zeta}\right)\right)
$$

Finally, the comprehensive concordance/discordance index $\phi^{\rho}$ for the $\rho^{\text {th }}$ permutation can be defined as follows:

$$
\phi^{\rho}=\sum_{x_{\xi}, x_{\zeta} \in X} \phi^{\rho}\left(x_{\xi}, x_{\zeta}\right)=\sum_{x_{\xi}, x_{\zeta} \in X} \sum_{j=1}^{n}\left(w_{j} \phi_{j}^{\rho}\left(x_{\xi}, x_{\zeta}\right)\right) \text {. }
$$

It is found that the more the comprehensive concordance/discordance index value is, the better the ranking order of alternatives is. Therefore, we can determine the optimal ranking order of alternatives by comparing the values $\phi^{\rho}$ of each permutation $P_{\rho}$.
Based on the above analysis, the decision processes of the proposed interval-valued q-rung orthopair fuzzy QUALIFLEX method can be summarized as follows.

Step 1. Formulate interval-valued q-rung orthopair fuzzy MAGDM problem and identify the interval-valued q-rung orthopair fuzzy decision matrix, and transform the decision matrix $A^{(k)}$ into a standard matrix $R^{(k)}(k=1,2, \ldots, t)$ by using (39) and (40).

Step 2. Determine the weights of experts by using (46).

Step 3. Integrate the individual decision matrices $R^{(k)}=\left(r_{i j}^{(k)}\right)_{m \times n}(k=1,2, \ldots, t)$ into to the collective decision matrix $R=\left(r_{i j}\right)_{m \times n}$ by $(47)$.

Step 4. List all of the possible $m$ ! permutations that should be tested in the next steps. Let $P_{\rho}$ denote the $\rho^{\text {th }}$ permutation using (48).

Step 5. Calculate the concordance/discordance index $\phi_{j}^{\rho}\left(x_{\xi}, x_{\zeta}\right)$ using (49).

Step 6. Calculate the weighted concordance/discordance indices $\phi^{\rho}\left(x_{\xi}, x_{\zeta}\right)$ using $(50)$.

Step 7. Calculate the comprehensive concordance/discordance index $\phi^{\rho}$ for permutation $P_{\rho}$ using (51).

Step 8. Determine the optimal ranking order of the alternatives. On the basis of the results in step 7, the permutation with the maximal comprehensive concordance/discordance index $\phi^{\rho}$ is the optimal ranking order of the alternatives; namely,

$$
P^{*}=\max _{\rho=1}^{m !}\left\{\phi^{\rho}\right\}
$$

The decision processes of the proposed interval-valued q-rung orthopair fuzzy QUALIFLEX method are described in Figure 1.

\section{Case Study on Health Management of Hypertensive Patients}

In order to improve the management efficiency of rural doctors, it is planned to develop the Department of Hypertension Daily Follow-up Early Warning Management. After research, it is determined that the warning colors are 5 types, $x_{i}(i=1,2,3,4,5): x_{1}$ means urgent handling and notifying residents to seek doctor and treatment immediately, shown in red; $x_{2}$ means urgent treatment is required and informing residents to seek doctor in time, shown in orange; $x_{3}$ means timely follow-up is needed to promote management, shown in yellow; $x_{4}$ means short-term need to promote services, shown in blue; $x_{5}$ means temporary no need to promote management services, shown in green. $c_{1}$ (measurement value of blood pressure), $c_{2}$ (cardiovascular disease), $c_{3}$ (family history of hypertension), and $c_{4}$ (obesity) 


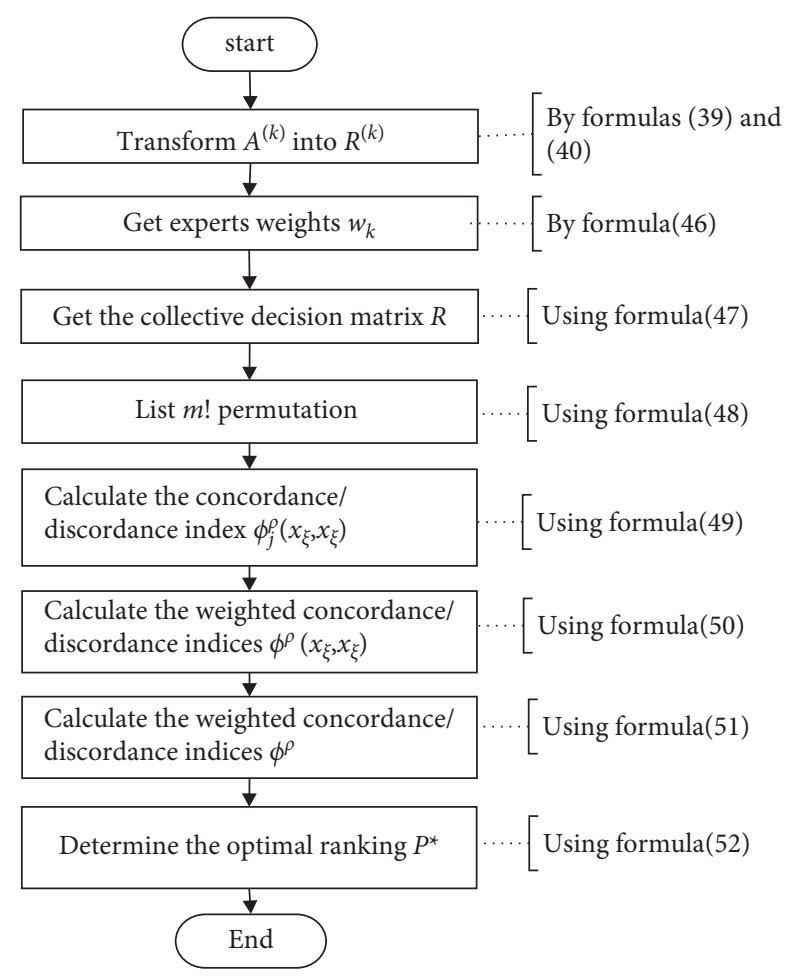

FIGURE 1: Frame diagram of interval-valued q-rung orthopair fuzzy QUALIFLEX method.

are used to express their attributes, and the weights of them are $w=(0.7,0.1,0.1,0.1)$.

In the absence of medication, for a 60-year-old male resident, a certain measurement value and related factors of collection are as follows: systolic blood pressure is $153 \mathrm{mmHg}$, dilation pressure is $98 \mathrm{mmHg}$, there are no related disease records, obesity, or family history of hypertension, the doctor has no follow-up records, the evaluation value given by four experts is expressed by q-ROFNs, because the cost type and benefit type do not require complicated calculations, and the amount of data is small, and the converted evaluation matrix is directly given in this paper. The evaluation values are given in Tables 1-4.

5.1. The Calculated Results by the Developed Method. We next use the developed method to identify the optimal alternative. Owing to the fact that the weights of experts are unknown in advance in this case, we first need to objectively determine the weights of experts. Using (41), the positive ideal group opinion with the form of interval-valued q-rung orthopair fuzzy matrix $\mathrm{CR}^{+}$is determined in Table 5 .

The negative ideal group opinion is obtained by (42) and the calculated results are listed in Table 6.

Similarly, using (43) and (44), the negative ideal right group opinion and the negative ideal left group opinion are obtained, and the calculated results are listed in Tables 7 and 8.

Then, using (45), the closeness indices of experts are obtained as follows:

$$
\mathrm{CI}_{k}=[0.9037,0.8084,0.8986,0.9014] \text {. }
$$

On the basis of the closeness indices of experts, the expert weight vector is obtained by (46) as

$$
\omega_{k}=[0.2573,0.2302,0.2559,0.2566] \text {. }
$$

Furthermore, we use the IVq - ROFDWA operator (47) to integrate the individual decision matrices into to the collective decision matrix and the calculated results are listed in Table 9.

Using (49), we can calculate the concordance/discordance index $\phi_{j}^{\rho}\left(x_{\xi}, x_{\zeta}\right) \quad(\rho=1,2, \ldots, 120 ; j=1,2,3,4 ; \xi$, $\zeta=1,2, \ldots, 5)$, and to save the space we only provide the calculated results of the concordance/discordance index $\phi_{j}^{\rho}\left(x_{\xi}, x_{\zeta}\right)$ under $\rho=1$. These calculated results are listed in Table 10 .

Then, the weighted concordance/discordance indices $\phi^{\rho}\left(x_{\xi}, x_{\zeta}\right)$ can be obtained by using (50). The calculated results are listed in Table 11 .

Finally, we can calculate the comprehensive concordance/discordance index $\phi^{\rho}$ for permutation $P_{\rho}$ using (51). The calculated results are listed in Table 12.

Using (52), the permutation with the maximal value of the comprehensive concordance/discordance indices is identified as $\phi^{\rho}=3.31$, and the corresponding permutation of alternatives is $x_{3}>x_{2}>x_{1}>x_{4}>x_{5}$. Thus, $x_{3}$ is the optimal alternative. This plan is a yellow warning, which means that timely follow-up with the doctor is needed and the patient should seek medical attention immediately. This decision result is basically consistent with medical experts.

5.2. Sensitive Analysis of Parameters. In order to reflect the influence of parameters on the final sorting results, we next conduct a sensitivity analysis by modifying the values of parameters $q$ and $\varphi$. We first calculate scores of alternatives by changing the values of parameter $q$ from 3 to 7 for verifying the influence of the change of $q$ in the q-rung orthogonal fuzzy environment on the group decision-making method. The calculation results are shown in Figure 2. It can be verified that the results of group decision-making remain unchanged when the value of parameter $q$ is changed from 3 to 7 .

Then, we modify simultaneously the values of $q$ and $\varphi$; namely, the value of $q$ is changed from 3 to 6 , and the value of $\varphi$ is changed from 3 to 6 ; the calculated results are shown in Figure 3. It can be seen from Figure 3 that the ranking results of alternatives do not change with the change of the values of parameters $q$ and $\varphi$. From the above analysis, the ranking results of alternatives will not be changed although they are different trends with the change of $q$ and $\varphi$; the final results will not be affected in the group decision-making method proposed in this paper. The feasibility and effectiveness of this method are verified.

5.3. Comparative Analysis. To show the advantage of the developed method, on the one hand, we next conduct a comparison analysis with various aggregating operators- 
Table 1: Decision matrix given by Expert $1 R^{(1)}$.

\begin{tabular}{ccccc}
\hline & $c_{1}$ & $c_{2}$ & $c_{3}$ & $c_{4}$ \\
\hline$x_{1}$ & $([0.7,0.8],[0.2,0.3])$ & $([0.5,0.6],[0.6,0.7])$ & $([0.8,0.9],[0.1,0.2])$ & $([0.8,0.95],[0.1,0.2])$ \\
$x_{2}$ & $([0.8,0.9],[0.1,0.2])$ & $([0.5,0.55],[0.4,0.5])$ & $([0.8,0.9],[0.1,0.15])$ & $([0.8,0.95],[0.1,0.2])$ \\
$x_{3}$ & $([0.9,0.95],[0.1,0.2])$ & $([0.5,0.6],[0.3,0.4])$ & $([0.8,0.85],[0.2,0.3])$ & $([0.8,0.9],[0.1,0.2])$ \\
$x_{4}$ & $([0.6,0.7],[0.4,0.5])$ & $([0.4,0.5],[0.3,0.4])$ & $([0.75,0.8],[0.2,0.3])$ & $([0.7,0.8],[0.2,0.3])$ \\
$x_{5}$ & $([0.1,0.2],[0.8,0.9])$ & $([0.2,0.3],[0.5,0.6])$ & $([0.7,0.85],[0.3,0.4])$ & $([0.6,0.7],[0.2,0.3])$ \\
\hline
\end{tabular}

TABle 2: Decision matrix given by Expert $2 R^{(2)}$.

\begin{tabular}{ccccc}
\hline & $c_{1}$ & $c_{2}$ & $c_{3}$ & $c_{4}$ \\
\hline$x_{1}$ & $([0.6,0.7],[0.2,0.3])$ & $([0.6,0.65],[0.4,0.5])$ & $([0.7,0.8],[0.1,0.2])$ & $([0.6,0.65],[0.2,0.3])$ \\
$x_{2}$ & $([0.85,0.9],[0.1,0.15])$ & $([0.5,0.55],[0.4,0.5])$ & $([0.6,0.7],[0.1,0.15])$ & $([0.7,0.75],[0.1,0.2])$ \\
$x_{3}$ & $([0.9,0.95],[0.1,0.15])$ & $([0.5,0.6],[0.3,0.4])$ & $([0.5,0.6],[0.2,0.3])$ & $([0.45,0.5],[0.2,0.3])$ \\
$x_{4}$ & $([0.2,0.3],[0.7,0.8])$ & $([0.4,0.5],[0.3,0.4])$ & $([0.70,0.80],[0.2,0.3])$ & $([0.50,0.6],[0.1,0.2])$ \\
$x_{5}$ & $([0.1,0.2],[0.8,0.95])$ & $([0.2,0.3],[0.5,0.6])$ & $([0.7,0.80],[0.3,0.4])$ & $([0.5,0.6],[0.1,0.2])$ \\
\hline
\end{tabular}

Table 3: Decision matrix given by Expert $3 R^{(3)}$.

\begin{tabular}{ccccc}
\hline & $c_{1}$ & $c_{2}$ & $c_{3}$ & $c_{4}$ \\
\hline$x_{1}$ & $([0.7,0.9],[0.2,0.3])$ & $([0.5,0.6],[0.4,0.5])$ & $([0.8,0.9],[0.1,0.2])$ & $([0.7,0.8],[0.1,0.2])$ \\
$x_{2}$ & $([0.7,0.9],[0.1,0.2])$ & $([0.5,0.55],[0.4,0.5])$ & $([0.8,0.9],[0.1,0.3])$ & $([0.7,0.90],[0.1,0.2])$ \\
$x_{3}$ & $([0.8,0.95],[0.1,0.2])$ & $([0.6,0.7],[0.3,0.4])$ & $([0.8,0.9],[0.1,0.3])$ & $([0.8,0.9],[0.1,0.2])$ \\
$x_{4}$ & $([0.5,0.6],[0.4,0.5])$ & $([0.5,0.6],[0.3,0.4])$ & $([0.75,0.85],[0.1,0.3])$ & $([0.7,0.8],[0.3,0.4])$ \\
$x_{5}$ & $([0.1,0.2],[0.8,0.9])$ & $([0.4,0.5],[0.7,0.8])$ & $([0.75,0.8],[0.2,0.4])$ & $([0.75,0.85],[0.1,0.2])$ \\
\hline
\end{tabular}

TABle 4: Decision matrix given by Expert $4 R^{(4)}$.

\begin{tabular}{ccccc}
\hline & $c_{1}$ & $c_{2}$ & $c_{3}$ & $c_{4}$ \\
\hline$x_{1}$ & $([0.7,0.8],[0.2,0.3])$ & $([0.55,0.65],[0.3,0.4])$ & $([0.7,0.9],[0.15,0.2])$ & $([0.70,0.80],[0.1,0.2])$ \\
$x_{2}$ & $([0.8,0.9],[0.15,0.2])$ & $([0.55,0.6],[0.4,0.5])$ & $([0.7,0.85],[0.1,0.15])$ & $([0.70,0.80],[0.2,0.3])$ \\
$x_{3}$ & $([0.8,0.9],[0.15,0.2])$ & $([0.5,0.6],[0.3,0.4])$ & $([0.7,0.9],[0.1,0.3])$ & $([0.8,0.9],[0.1,0.2])$ \\
$x_{4}$ & $([0.6,0.7],[0.6,0.7])$ & $([0.4,0.6],[0.6,0.7])$ & $([0.6,0.8],[0.2,0.3])$ & $([0.7,0.8],[0.1,0.3])$ \\
$x_{5}$ & $([0.1,0.2],[0.85,0.9])$ & $([0.4,0.5],[0.75,0.8])$ & $([0.7,0.8],[0.3,0.4])$ & $([0.7,0.8],[0.1,0.2])$ \\
\hline
\end{tabular}

TABLE 5: The positive ideal group opinion matrix $\mathrm{CR}^{+}$.

\begin{tabular}{ccccc}
\hline & $c_{1}$ & $c_{2}$ & $c_{3}$ & $c_{4}$ \\
\hline$x_{1}$ & $([0.68,0.85],[0.20,0.30])$ & $([0.55,0.63],[0.50,0.60])$ & $([0.77,0.89],[0.12,0.20])$ & $([0.73,0.91],[0.16,0.25])$ \\
$x_{2}$ & $([0.81,0.90],[0.12,0.19])$ & $([0.52,0.57],[0.40,0.50])$ & $([0.76,0.88],[0.10,0.24])$ & $([0.74,0.92],[0.16,0.25])$ \\
$x_{3}$ & $([0.88,0.94],[0.12,0.19])$ & $([0.54,0.64],[0.30,0.40])$ & $([0.76,0.88],[0.18,0.30])$ & $([0.78,0.89],[0.16,0.25])$ \\
$x_{4}$ & $([0.51,0.61],[0.66,0.76])$ & $([0.44,0.56],[0.50,0.60])$ & $([0.72,0.82],[0.19,0.30])$ & $([0.68,0.78],[0.24,0.34])$ \\
$x_{5}$ & $([0.10,0.20],[0.82,0.93])$ & $([0.36,0.45],[0.68,0.76])$ & $([0.72,0.82],[0.29,0.40])$ & $([0.69,0.79],[0.16,0.25])$ \\
\hline
\end{tabular}

TABLE 6: The negative ideal group opinion matrix $\mathrm{CR}^{-}$.

\begin{tabular}{ccccc}
\hline & $c_{1}$ & $c_{2}$ & $c_{3}$ & $c_{4}$ \\
\hline$x_{1}$ & $([0.20,0.30],[0.68,0.85])$ & $([0.50,0.60],[0.55,0.63])$ & $([0.12,0.20],[0.77,0.89])$ & $([0.16,0.25],[0.73,0.91])$ \\
$x_{2}$ & $([0.12,0.19],[0.81,0.90])$ & $([0.40,0.50],[0.52,0.57])$ & $([0.10,0.24],[0.76,0.88])$ & $([0.16,0.25],[0.74,0.92])$ \\
$x_{3}$ & $([0.12,0.19],[0.88,0.94])$ & $([0.30,0.40],[0.54,0.64])$ & $([0.18,0.30],[0.76,0.88])$ & $([0.16,0.25],[0.78,0.89])$ \\
$x_{4}$ & $([0.66,0.76],[0.51,0.61])$ & $([0.50,0.60],[0.44,0.56])$ & $([0.19,0.30],[0.72,0.82])$ & $([0.24,0.34],[0.68,0.78])$ \\
$x_{5}$ & $([0.82,0.93],[0.10,0.20])$ & $([0.68,0.76],[0.36,0.45])$ & $([0.29,0.40],[0.72,0.82])$ & $([0.16,0.25],[0.69,0.79])$ \\
\hline
\end{tabular}


TABLE 7: The negative ideal right group opinion matrix $\mathrm{Ir}^{+}$.

\begin{tabular}{ccccc}
\hline & $c_{1}$ & $c_{2}$ & $c_{3}$ & $c_{4}$ \\
\hline$x_{1}$ & $([0.70,0.90],[0.20,0.30])$ & $([0.60,0.65],[0.60,0.70])$ & $([0.80,0.90],[0.15,0.20])$ & $([0.80,0.95],[0.20,0.30])$ \\
$x_{2}$ & $([0.85,0.90],[0.15,0.20])$ & $([0.55,0.60],[0.40,0.50])$ & $([0.80,0.90],[0.10,0.30])$ & $([0.80,0.95],[0.20,0.30])$ \\
$x_{3}$ & $([0.90,0.95],[0.15,0.20])$ & $([0.60,0.70],[0.30,0.40])$ & $([0.80,0.90],[0.20,0.30])$ & $([0.80,0.90],[0.20,0.30])$ \\
$x_{4}$ & $([0.60,0.70],[0.70,0.80])$ & $([0.50,0.60],[0.60,0.70])$ & $([0.75,0.85],[0.20,0.30])$ & $([0.70,0.80],[0.30,0.40])$ \\
$x_{5}$ & $([0.10,0.20],[0.85,0.95])$ & $([0.40,0.50],[0.75,0.80])$ & $([0.75,0.85],[0.30,0.40])$ & $([0.75,0.85],[0.20,0.30])$ \\
\hline
\end{tabular}

TABLE 8: The negative ideal left group opinion matrix $I r^{-}$.

\begin{tabular}{ccccc}
\hline & $c_{1}$ & $c_{2}$ & $c_{3}$ & $c_{4}$ \\
\hline$x_{1}$ & $([0.60,0.70],[0.20,0.30])$ & $([0.50,0.60],[0.30,0.40])$ & $([0.70,0.80],[0.10,0.20])$ & $([0.60,0.65],[0.10,0.20])$ \\
$x_{2}$ & $([0.70,0.90],[0.10,0.15])$ & $([0.50,0.55],[0.40,0.50])$ & $([0.60,0.70],[0.10,0.15])$ & $([0.70,0.75],[0.10,0.20])$ \\
$x_{3}$ & $([0.80,0.90],[0.10,0.15])$ & $([0.50,0.60],[0.30,0.40])$ & $([0.50,0.60],[0.10,0.30])$ & $([0.45,0.50],[0.10,0.20])$ \\
$x_{4}$ & $([0.20,0.30],[0.40,0.50])$ & $([0.40,0.50],[0.30,0.40])$ & $([0.60,0.80],[0.10,0.30])$ & $([0.50,0.60],[0.10,0.20])$ \\
$x_{5}$ & $([0.10,0.20],[0.80,0.90])$ & $([0.20,0.30],[0.50,0.60])$ & $([0.70,0.80],[0.20,0.40])$ & $([0.50,0.60],[0.10,0.20])$ \\
\hline
\end{tabular}

TABLe 9: The collective decision matrix $R$.

\begin{tabular}{ccccc}
\hline & $c_{1}$ & $c_{2}$ & $c_{3}$ & $c_{4}$ \\
\hline$x_{1}$ & $([0.69,0.85],[0.20,0.30])$ & $([0.55,0.63],[0.51,0.61])$ & $([0.77,0.89],[0.12,0.20])$ & $([0.74,0.91],[0.16,0.25])$ \\
$x_{2}$ & $([0.81,0.90],[0.12,0.19])$ & $([0.52,0.57],[0.40,0.50])$ & $([0.76,0.88],[0.10,0.24])$ & $([0.74,0.92],[0.16,0.25])$ \\
$x_{3}$ & $([0.87,0.94],[0.12,0.19])$ & $([0.54,0.64],[0.30,0.40])$ & $([0.76,0.88],[0.18,0.30])$ & $([0.78,0.89],[0.16,0.25])$ \\
$x_{4}$ & $([0.51,0.61],[0.66,0.76])$ & $([0.44,0.57],[0.50,0.60])$ & $([0.72,0.82],[0.19,0.30])$ & $([0.68,0.78],[0.24,0.34])$ \\
$x_{5}$ & $([0.10,0.20],[0.82,0.92])$ & $([0.36,0.46],[0.68,0.76])$ & $([0.72,0.82],[0.29,0.40])$ & $([0.69,0.79],[0.16,0.25])$ \\
\hline
\end{tabular}

TABLE 10: The results of the concordance/discordance index under $P_{1}$.

\begin{tabular}{|c|c|c|c|c|c|}
\hline$P_{1}$ & $\phi_{j}^{1}\left(x_{1}, x_{2}\right)$ & $\phi_{j}^{1}\left(x_{1}, x_{3}\right)$ & $\phi_{j}^{1}\left(x_{1}, x_{4}\right)$ & $\phi_{j}^{1}\left(x_{1}, x_{5}\right)$ & $\phi_{j}^{2}\left(x_{2}, x_{3}\right)$ \\
\hline$c_{1}$ & -0.16 & -0.27 & 0.35 & 0.65 & -0.11 \\
\hline$c_{2}$ & -0.13 & -0.14 & 0.08 & 0.2 & -0.06 \\
\hline$c_{3}$ & 0.02 & 0.02 & 0.12 & 0.12 & 0.01 \\
\hline$c_{4}$ & -0.01 & -0.06 & 0.18 & 0.17 & 0.07 \\
\hline$P_{1}$ & $\phi_{j}^{2}\left(x_{2}, x_{4}\right)$ & $\phi_{j}^{2}\left(x_{2}, x_{5}\right)$ & $\phi_{j}^{2}\left(x_{3}, x_{4}\right)$ & $\phi_{j}^{2}\left(x_{3}, x_{5}\right)$ & $\phi_{j}^{2}\left(x_{4}, x_{5}\right)$ \\
\hline$c_{1}$ & 0.45 & 0.66 & 0.56 & 0.74 & 0.30 \\
\hline$c_{2}$ & 0.08 & 0.28 & 0.12 & 0.33 & 0.21 \\
\hline$c_{3}$ & 0.10 & 0.10 & 0.10 & 0.10 & 0.03 \\
\hline$c_{4}$ & 0.20 & 0.18 & 0.20 & 0.18 & -0.02 \\
\hline
\end{tabular}

Table 11: The results of the weighted concordance/discordance index under $P_{1}$.

\begin{tabular}{|c|c|c|c|c|c|}
\hline$P_{1}$ & $\phi_{j}^{1}\left(x_{1}, x_{2}\right)$ & $\phi_{j}^{1}\left(x_{1}, x_{3}\right)$ & $\phi_{j}^{1}\left(x_{1}, x_{4}\right)$ & $\phi_{j}^{1}\left(x_{1}, x_{5}\right)$ & $\phi_{j}^{2}\left(x_{2}, x_{3}\right)$ \\
\hline$c_{1}$ & -0.1112 & -0.1913 & 0.2420 & 0.4533 & -0.0800 \\
\hline$c_{2}$ & -0.0131 & -0.0139 & 0.0082 & 0.0197 & -0.0057 \\
\hline$c_{3}$ & 0.0021 & 0.0021 & 0.0118 & 0.0118 & 0.0009 \\
\hline$c_{4}$ & -0.0013 & -0.0059 & 0.0185 & 0.0169 & 0.0072 \\
\hline$P_{1}$ & $\phi_{j}^{2}\left(x_{2}, x_{4}\right)$ & $\phi_{j}^{2}\left(x_{2}, x_{5}\right)$ & $\phi_{j}^{2}\left(x_{3}, x_{4}\right)$ & $\phi_{j}^{2}\left(x_{3}, x_{5}\right)$ & $\phi_{j}^{2}\left(x_{4}, x_{5}\right)$ \\
\hline$c_{1}$ & 0.3153 & 0.4625 & 0.3953 & 0.5180 & 0.2113 \\
\hline$c_{2}$ & 0.0076 & 0.0282 & 0.0125 & 0.0331 & 0.0206 \\
\hline$c_{3}$ & 0.0098 & 0.0098 & 0.0098 & 0.0098 & 0.0027 \\
\hline$c_{4}$ & 0.0197 & 0.0181 & 0.0195 & 0.0179 & -0.0019 \\
\hline
\end{tabular}


TABLE 12: The results of the concordance/discordance index.

\begin{tabular}{|c|c|c|c|c|c|c|c|c|c|}
\hline$P_{1}$ & $P_{2}$ & $P_{3}$ & $P_{4}$ & $P_{5}$ & $P_{6}$ & $P_{7}$ & $P_{8}$ & $P_{9}$ & $P_{10}$ \\
\hline 2.49 & 2.03 & 1.62 & 0.46 & 0.87 & -0.01 & 2.65 & 2.18 & 1.94 & 0.91 \\
\hline$P_{11}$ & $P_{12}$ & $P_{13}$ & $P_{14}$ & $P_{15}$ & $P_{16}$ & $P_{17}$ & $P_{18}$ & $P_{19}$ & $P_{20}$ \\
\hline 1.14 & 0.44 & 0.91 & -0.24 & 1.07 & 0.03 & -1.28 & -1.13 & -0.17 & -1.04 \\
\hline$P_{21}$ & $P_{22}$ & $P_{23}$ & $P_{24}$ & $P_{25}$ & $P_{26}$ & $P_{27}$ & $P_{28}$ & $P_{29}$ & $P_{30}$ \\
\hline-0.01 & -0.72 & -1.75 & -1.59 & 2.74 & 2.27 & 1.86 & 0.71 & 1.12 & 0.24 \\
\hline$P_{31}$ & $P_{32}$ & $P_{33}$ & $P_{34}$ & $P_{35}$ & $P_{36}$ & $P_{37}$ & $P_{38}$ & $P_{39}$ & $P_{40}$ \\
\hline 3.16 & 2.69 & 2.60 & 1.59 & 1.69 & 1.13 & 1.30 & 0.15 & 1.72 & 0.72 \\
\hline$P_{41}$ & $P_{42}$ & $P_{43}$ & $P_{44}$ & $P_{45}$ & $P_{46}$ & $P_{47}$ & $P_{48}$ & $P_{49}$ & $P_{50}$ \\
\hline-0.86 & -0.44 & 0.11 & -0.76 & 0.53 & -0.03 & -1.32 & -0.91 & 3.07 & 2.60 \\
\hline$P_{51}$ & $P_{52}$ & $P_{53}$ & $P_{54}$ & $P_{55}$ & $P_{56}$ & $P_{57}$ & $P_{58}$ & $P_{59}$ & $P_{60}$ \\
\hline 2.36 & 1.32 & 1.56 & 0.86 & 3.31 & 2.85 & 2.75 & 1.75 & 1.84 & 1.28 \\
\hline$P_{61}$ & $P_{62}$ & $P_{63}$ & $P_{64}$ & $P_{65}$ & $P_{66}$ & $P_{67}$ & $P_{68}$ & $P_{69}$ & $P_{70}$ \\
\hline 1.80 & 0.76 & 2.05 & 1.04 & -0.24 & 0.01 & 0.56 & -0.15 & 0.81 & 0.24 \\
\hline$P_{71}$ & $P_{72}$ & $P_{73}$ & $P_{74}$ & $P_{75}$ & $P_{76}$ & $P_{77}$ & $P_{78}$ & $P_{79}$ & $P_{80}$ \\
\hline-0.71 & -0.46 & 0.35 & -0.81 & 0.51 & -0.53 & -1.84 & -1.69 & 0.60 & -0.56 \\
\hline$P_{81}$ & $P_{82}$ & $P_{83}$ & $P_{84}$ & $P_{85}$ & $P_{86}$ & $P_{87}$ & $P_{88}$ & $P_{89}$ & $P_{90}$ \\
\hline 1.02 & 0.01 & -1.56 & -1.14 & 0.92 & -0.11 & 1.17 & 0.17 & -1.12 & -0.87 \\
\hline$P_{91}$ & $P_{92}$ & $P_{93}$ & $P_{94}$ & $P_{95}$ & $P_{96}$ & $P_{97}$ & $P_{98}$ & $P_{99}$ & $P_{100}$ \\
\hline-2.85 & -2.69 & -2.60 & -2.18 & -2.27 & -2.03 & -1.17 & -2.05 & -1.02 & -1.72 \\
\hline$P_{101}$ & $P_{102}$ & $P_{103}$ & $P_{104}$ & $P_{105}$ & $P_{106}$ & $P_{107}$ & $P_{108}$ & $P_{109}$ & $P_{110}$ \\
\hline-2.75 & -2.60 & -0.92 & -1.80 & -0.51 & -1.07 & -2.36 & -1.94 & -0.60 & -1.30 \\
\hline$P_{111}$ & $P_{112}$ & $P_{113}$ & $P_{114}$ & $P_{115}$ & $P_{116}$ & $P_{117}$ & $P_{118}$ & $P_{119}$ & $P_{120}$ \\
\hline-0.35 & -0.91 & -1.86 & -1.62 & -3.31 & -3.16 & -3.07 & -2.65 & -2.74 & -2.49 \\
\hline
\end{tabular}



FIgURE 2: Sensitivity analysis of parameter $q$.

based decision-making methods proposed in [39, 40]. Therefore, we next use the IVPFWA, IVPFOWA, and IVPFWG operators proposed in $[39,40]$ to obtain the rankings of alternatives. It is worthy to point out that these operators proposed in $[39,40]$ can only be used to calculate the IVPFNs but failed to deal with the IVq-ROFNs. Thus, in this comparison analysis, we set the value of the parameter as 2 and all IVq-ROFNs data in the above health management of hypertensive patients are transformed IVPFNs data. Then, the calculated results are obtained and shown in Table 13.
It is noticed that the obtained ranking orders of alternatives are the same. Nevertheless, the information loss always happens in the process of information aggregation [41] when these aggregating operators-based decisionmaking methods $[39,40]$ are used. Meanwhile our developed method not only is capable of dealing with the situations where the weight information of the experts is unknown but also can reduce the information loss.

On the other hand, we conduct an analysis to compare between our method and the IVq-ROF MULTIMOORA 


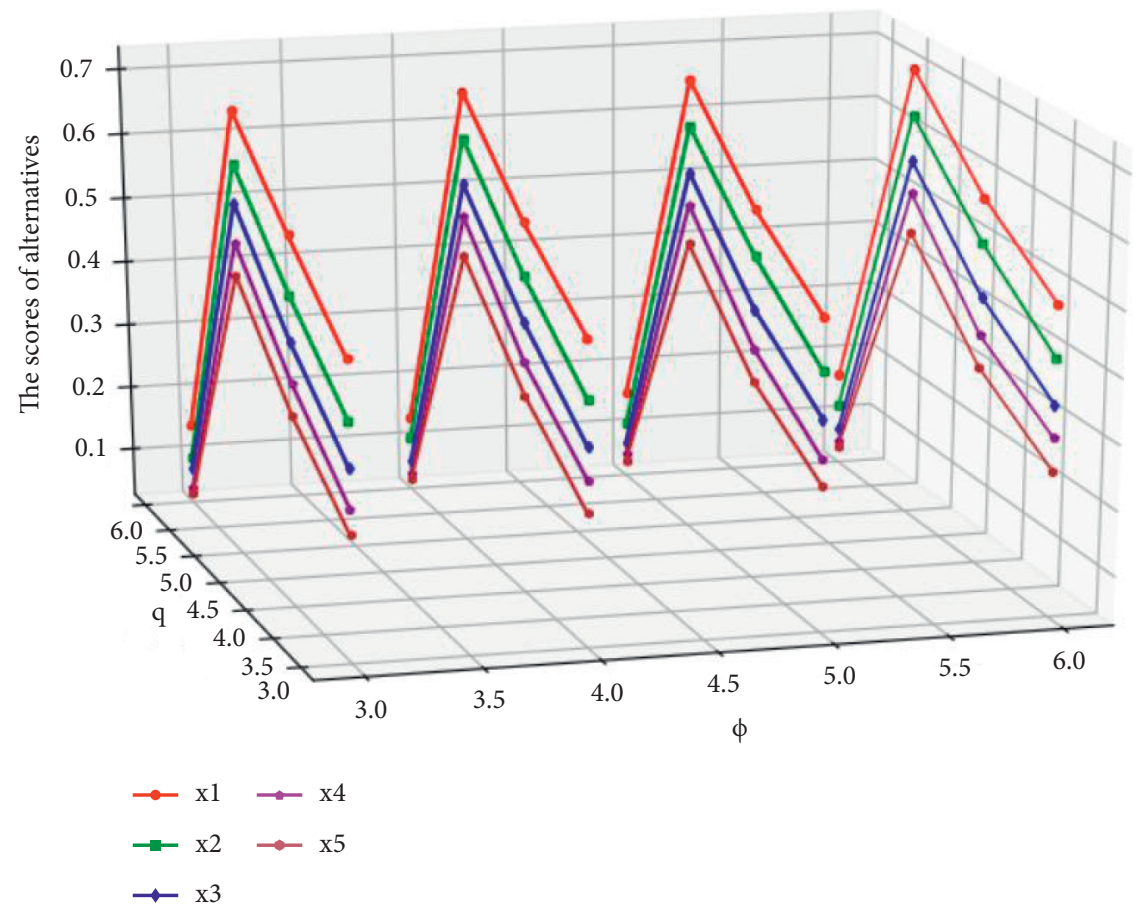

FIGURe 3: Sensitivity analysis of parameters $q$ and $\varphi$.

TABLE 13: Comparison analysis of the calculation results obtained by different methods.

\begin{tabular}{|c|c|c|c|c|c|c|}
\hline \multirow{2}{*}{ Methods } & \multicolumn{5}{|c|}{ Ranking scores of alternatives } & \multirow{2}{*}{ Rankings of alternatives } \\
\hline & $x 1$ & $x 2$ & $x 3$ & $x 4$ & $x 5$ & \\
\hline IVPFWA & 0.44 & 0.61 & 0.69 & -0.04 & -0.44 & $x_{3}>x_{2}>x_{1}>x_{4}>x_{5}$ \\
\hline IVPFOWA & 0.37 & 0.45 & 0.49 & 0.08 & -0.19 & $x_{3}>x_{2}>x_{1}>x_{4}>x_{5}$ \\
\hline IVPFWG & 0.23 & 0.32 & 0.37 & -0.24 & -0.63 & $x_{3}>x_{2}>x_{1}>x_{4}>x_{5}$ \\
\hline IVq-ROFDWA & 0.97 & 1.27 & 1.48 & -0.08 & -0.70 & $x_{3}>x_{2}>x_{1}>x_{4}>x_{5}$ \\
\hline IVq-ROF QUALIFLEX & - & - & - & - & - & $x_{3}>x_{2}>x_{1}>x_{4}>x_{5}$ \\
\hline
\end{tabular}

method [42]. According to the IVq-ROF MULTIMOORA method, we first determine the ranking of alternatives based on the ratio system by using the following equation:

$$
\begin{aligned}
& \tilde{y}^{*}\left(r_{i}\right)=q-\operatorname{RIVOFWA}\left(r_{i 1}, r_{i 2}, \ldots, r_{i n}\right)
\end{aligned}
$$

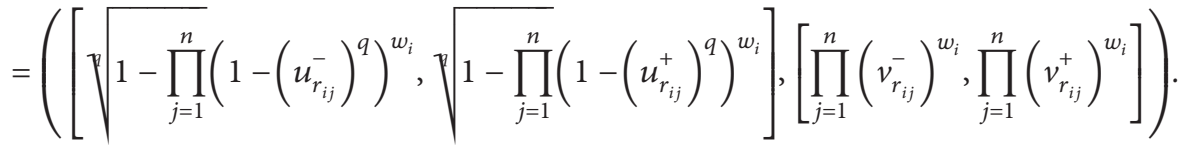

Then, the scores of alternatives are obtained as $x_{1}=0.53$, $x_{2}=0.66, x_{3}=0.73, x_{4}=0.04$, and $x_{5}=-0.26$, and the ranking of alternatives is $x_{3}>x_{2}>x_{1}>x_{4}>x_{5}$.
Next, we determine the ranking of alternatives based on the reference point approach by using the following equation:

$$
d\left(r_{i}, r^{*}\right)=\sum_{j=1}^{n} \frac{1}{4}\left(\left|\left(u_{r_{i j}}^{-}\right)^{q}-1\right|+\left|\left(u_{r_{i j}}^{+}\right)^{q}-1\right|+\left|\left(v_{r_{i j}}^{-}\right)^{q}-0\right|+\left|\left(v_{r_{i j}}^{+}\right)^{q}-0\right|+\left|\left(\pi_{r_{i j}}^{-}\right)^{q}-0\right|+\left|\left(\pi_{r_{i j}}^{+}\right)^{q}-0\right|\right) .
$$


The ideal distances of alternatives from $([1,1],[0,0])$ are determined as $x_{1}=0.63, x_{2}=0.49, x_{3}=0.34, x_{4}=0.91$, and $x_{5}=0.93$, and the ranking of alternatives is $x_{3}>x_{2}>x_{1}>x_{4}>x_{5}$.
Finally, we determine the ranking of alternatives based on the full multiplicative form by using the following equation:

$$
\begin{aligned}
& \widetilde{U}^{*}\left(r_{i}\right)=q-\operatorname{RIVOFWG}\left(r_{i 1}, r_{i 2}, \ldots, r_{i n}\right)
\end{aligned}
$$

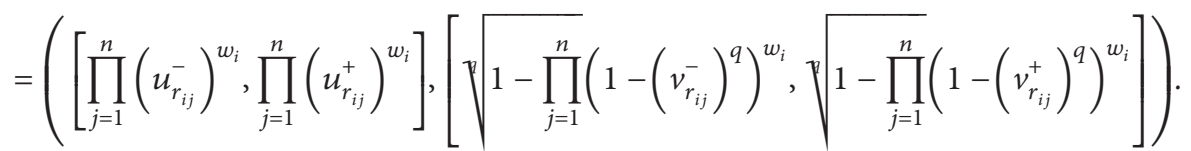

The scores of alternatives are got as $x_{1}=0.39, x_{2}=0.46$, $x_{3}=0.53, x_{4}=-0.29$, and $x_{5}=-0.74$, and, the ranking of alternatives is $x_{3}>x_{2}>x_{1}>x_{4}>x_{5}$. According to the dominance theory, the ranking of alternatives is $x_{3}>x_{2}>x_{1}>x_{4}>x_{5}$. It is noticed that the ranking orders of alternatives obtained by our developed method and by the IVq-ROF MULTIMOORA method [42] are the same, which further confirms the effectiveness of our developed method.

\section{Conclusion}

In this paper, we have developed an interval-valued q-rung orthopair fuzzy QUALIFLEX decision analysis method with Dombi operators. We have first defined interval-valued q-rung orthopair fuzzy Dombi operational laws according to $\mathrm{t}$-conorms and $\mathrm{t}$-norms algorithms of the Dombi operations. Second, based on the developed interval-valued q-rung orthopair fuzzy Dombi operational laws, we have proposed the IVq-ROFDWA operator and studied its proprieties. Third, with the help of IVq-ROFDWA operator, we have determined the ideal group opinions and constructed a new weight-determining method for identifying the weights of experts. The biggest advantage of the weight-determining method is that it can make sure that each expert's opinion is consistent with the group opinion to the greatest extent. Furthermore, we have proposed a new interval-valued q-rung orthopair fuzzy distance measure. With the help of the developed distance measure, we have defined the concept of the interval-valued q-rung orthopair fuzzy concordance/ discordance index. By calculating the comprehensive concordance/discordance index under each permutation, the final ranking orders of alternatives have been identified. The algorithm of interval-valued q-rung orthopair fuzzy QUALIFLEX decision analysis method has been introduced for addressing the complex MAGDM problems with IVqROFSs. Apparently, our developed method does not need to provide expert weights and attribute weights in advance, so it can improve the decision-making efficiency and save decision cost. Nevertheless, our developed method is only applicable to the case of a small number of experts and a small number of schemes, since it needs to traverse the ranking of all schemes in the decision-making process. With the increasing complexity of the system and the rapid growth of uncertainty, it is necessary to combine big data and artificial intelligence to deal with complex problems in the decision- making process. For this reason, we will study the group of complex systems of big data decision problems on the basis of the developed method. On the other hand, in real-life decision processes, decision-makers often give preference information between schemes and provide a judgment matrix instead of a decision matrix according to their own experience and knowledge. Therefore, group decisionmaking methods based on interval-valued q-rung orthopair fuzzy preference relations are our research focus.

\section{Data Availability}

The data used to support the findings of this study are included in the article.

\section{Conflicts of Interest}

The authors declare that they have no conflicts of interest.

\section{Acknowledgments}

This work was supported in part by the National Natural Science Foundation of China under Grants 71901112, 71661010, and 61363075, in part by the Department of Shenzhen Local Science and Technology Development under Grant 2021Szvup052, in part by the Natural Science Foundation of Jiangxi Province of China under Grants 20202BAB202006 and 20202BAA208012, in part by Social Science Planning Project of Jiangxi Province of China under Grant 19GL16, and in part by Department of Education of Jiangxi Province of China under Grant GJJ180270.

\section{References}

[1] K. T. Atanassov, "Intuitionistic fuzzy sets," Fuzzy Sets and Systems, vol. 20, no. 1, pp. 87-96, 1986.

[2] R. R. Yager, "Pythagorean membership grades in multicriteria decision making," IEEE Transactions on Fuzzy Systems, vol. 22, no. 4, pp. 958-965, 2013.

[3] X. Zhang and $\mathrm{Z}$. Xu, "Extension of TOPSIS to multiple criteria decision making with Pythagorean fuzzy sets," International Journal of Intelligent Systems, vol. 29, no. 12, pp. 1061-1078, 2014.

[4] X. Zhang, "Multicriteria Pythagorean fuzzy decision analysis: a hierarchical QUALIFLEX approach with the closeness index-based ranking methods," Information Sciences, vol. 330, pp. 104-124, 2016. 
[5] R. R. Yager, "Generalized orthopair fuzzy sets," IEEE Transactions on Fuzzy Systems, vol. 25, no. 5, pp. 1222-1230, 2016.

[6] S. Zeng, S. M. Chen, and K. Y. Fan, "Interval-valued intuitionistic fuzzy multiple attribute decision making based on nonlinear programming methodology and TOPSIS method," Information Sciences, vol. 506, pp. 424-442, 2020.

[7] R. R. Yager and N. Alajlan, "Approximate reasoning with generalized orthopair fuzzy sets," Information Fusion, vol. 38, pp. 65-73, 2017.

[8] J. Gao, Z. Liang, and Z. Xu, “Additive integrals of \$ q \$-Rung orthopair fuzzy functions," IEEE Transactions on Cybernetics, vol. 50, no. 10, pp. 4406-4419, 2019.

[9] J. Gao, Z. Liang, J. Shang, and Z. Xu, "Continuities, derivatives, and differentials of q-rung orthopair fuzzy functions," IEEE Transactions on Fuzzy Systems, vol. 27, no. 8, pp. 1687-1699, 2018.

[10] X. Shu, Z. Ai, Z. Xu, and J. Ye, "Integrations of q-rung orthopair fuzzy continuous information," IEEE Transactions on Fuzzy Systems, vol. 27, no. 10, pp. 1974-1985, 2019.

[11] G. Wei, H. Gao, and Y. Wei, "Some q-rung orthopair fuzzy Heronian mean operators in multiple attribute decision making," International Journal of Intelligent Systems, vol. 33, no. 7, pp. 1426-1458, 2018.

[12] P. Liu, S. M. Chen, and P. Wang, "Multiple-attribute group decision-making based on q-rung orthopair fuzzy power maclaurin symmetric mean operators," IEEE Transactions on Systems, Man, and Cybernetics: Systems, vol. 50, no. 10, pp. 3741-3756, 2018.

[13] P. Liu and P. Wang, "Multiple-attribute decision-making based on Archimedean Bonferroni Operators of q-rung orthopair fuzzy numbers," IEEE Transactions on Fuzzy Systems, vol. 27, no. 5, pp. 834-848, 2018.

[14] H. Garg and S.-M. Chen, "Multiattribute group decision making based on neutrality aggregation operators of q-rung orthopair fuzzy sets," Information Sciences, vol. 517, pp. 427-447, 2020.

[15] Y. Xing, R. Zhang, Z. Zhou, and J. Wang, "Some q-rung orthopair fuzzy point weighted aggregation operators for multi-attribute decision making," Soft Computing, vol. 23, no. 22, Article ID 11649, 2019.

[16] X. Peng and L. Liu, "Information measures for q -rung orthopair fuzzy sets," International Journal of Intelligent Systems, vol. 34, no. 8, pp. 1795-1834, 2019.

[17] W. S. Du, "Minkowski-type distance measures for generalized orthopair fuzzy sets," International Journal of Intelligent Systems, vol. 33, no. 4, pp. 802-817, 2018.

[18] S. Zeng, Y. Hu, and X. Xie, "Q-rung orthopair fuzzy weighted induced logarithmic distance measures and their application in multiple attribute decision making," Engineering Applications of Artificial Intelligence, vol. 100, Article ID 104167, 2021.

[19] H. Liao, H. Zhang, C. Zhang, X. Wu, A. Mardani, and A. AlBarakat, "A q-rung orthopair fuzzy GLDS method for investment evaluation of BE angel capital in China," Technological and Economic Development of Economy, vol. 26, no. 1, pp. 103-134, 2020.

[20] D. Banerjee, B. Dutta, D. Guha, and L. Martínez, "SMAAQUALIFLEX methodology to handle multicriteria decisionmaking problems based on q-rung fuzzy set with hierarchical structure of criteria using bipolar Choquet integral," International Journal of Intelligent Systems, vol. 35, no. 3, pp. 401-431, 2020.
[21] B. P. Joshi, A. Singh, P. K. Bhatt, and K. S. Vaisla, "Interval valued q-rung orthopair fuzzy sets and their properties," Journal of Intelligent and Fuzzy Systems, vol. 35, no. 5, pp. 5225-5230, 2018.

[22] J. Wang, H. Gao, G. Wei, and Y. Wei, "Methods for multipleattribute group decision making with q-rung interval-valued orthopair fuzzy information and their applications to the selection of green suppliers," Symmetry, vol. 11, no. 1, p. 56, 2019.

[23] C. Jin, Y. Ran, and G. Zhang, "Interval-valued q-rung orthopair fuzzy FMEA application to improve risk evaluation process of tool changing manipulator," Applied Soft Computing, vol. 104, Article ID 107192, 2021.

[24] Y. Yang, Z. S. Chen, R. M. Rodríguez, P. Witold, and K. S. Chin, "Novel fusion strategies for continuous intervalvalued q-rung orthopair fuzzy information: a case study in quality assessment of SmartWatch appearance design," International Journal of Machine Learning and Cybernetics, pp. 1-24, 2021.

[25] Z. Yang and J. Chang, "A multi-attribute decision-makingbased site selection assessment algorithm for garbage disposal plant using interval q-rung orthopair fuzzy power Muirhead mean operator," Environmental Research, vol. 193, Article ID 110385, 2021.

[26] J. H. P. Paelinck, "Qualiflex: a flexible multiple-criteria method," Economics Letters, vol. 1, no. 3, pp. 193-197, 1978.

[27] J. C. Wang, C. Y. Tsao, and T. Y. Chen, "A likelihood-based QUALIFLEX method with interval type-2 fuzzy sets for multiple criteria decision analysis," Soft Computing, vol. 19, no. 8, pp. 2225-2243, 2015.

[28] T. Y. Chen, C. H. Chang, and J. F. L. Rachel, "The extended QUALIFLEX method for multiple criteria decision analysis based on interval type-2 fuzzy sets and applications to medical decision making," European Journal of Operational Research, vol. 226, no. 3, pp. 615-625, 2013.

[29] X. Zhang and Z. Xu, "Hesitant fuzzy QUALIFLEX approach with a signed distance-based comparison method for multiple criteria decision analysis," Expert Systems with Applications, vol. 42, no. 2, pp. 873-884, 2015.

[30] T. Y. Chen, "Interval-valued intuitionistic fuzzy QUALIFLEX method with a likelihood-based comparison approach for multiple criteria decision analysis," Information Sciences, vol. 261, pp. 149-169, 2014.

[31] Y. Liang, J. Qin, L. Martínez, and J. Liu, “A heterogeneous QUALIFLEX method with criteria interaction for multi-criteria group decision making," Information Sciences, vol. 512, pp. 1481-1502, 2020.

[32] J. Dombi, "A general class of fuzzy operators, the DeMorgan class of fuzzy operators and fuzziness measures induced by fuzzy operators," Fuzzy Sets and Systems, vol. 8, no. 2, pp. 149-163, 1982.

[33] C. Jana, G. Muhiuddin, and M. Pal, "Some Dombi aggregation of Q -rung orthopair fuzzy numbers in multiple-attribute decision making," International Journal of Intelligent Systems, vol. 34, no. 12, pp. 3220-3240, 2019.

[34] P. Liu, J. Liu, and S. M. Chen, "Some intuitionistic fuzzy Dombi Bonferroni mean operators and their application to multi-attribute group decision making," Journal of the Operational Research Society, vol. 69, no. 1, pp. 1-24, 2017.

[35] C. Jana, T. Senapati, M. Pal, and R. R. Yager, "Picture fuzzy Dombi aggregation operators: application to MADM process," Applied Soft Computing, vol. 74, pp. 99-109, 2019.

[36] X. Zhang, H. Liao, B. Xu, and M. Xiong, "A probabilistic linguistic-based deviation method for multi-expert qualitative 
decision making with aspirations," Applied Soft Computing, vol. 93, Article ID 106362, 2020.

[37] X. Zhang, Z. Xu, and H. Wang, "Heterogeneous multiple criteria group decision making with incomplete weight information: a deviation modeling approach," Information Fusion, vol. 25, pp. 49-62, 2015.

[38] S. P. Wan, G. L. Xu, F. Wang, and J. Y. Dong, "A new method for Atanassov's interval-valued intuitionistic fuzzy MAGDM with incomplete attribute weight information," Information Sciences, vol. 316, pp. 329-347, 2015.

[39] K. Rahman, A. Ali, and M. A. K. Sajjad, "Some interval-valued Pythagorean fuzzy weighted averaging aggregation operators and their application to multiple attribute decision making," Punjab University Journal of Mathematics, vol. 50, no. 2, 2020.

[40] K. Rahman and S. Abdullah, "Generalized interval-valued Pythagorean fuzzy aggregation operators and their application to group decision-making," Granular Computing, vol. 4, no. 1, pp. 15-25, 2019.

[41] Z. Xu and X. Zhang, "Hesitant fuzzy multi-attribute decision making based on TOPSIS with incomplete weight information," Knowledge-Based Systems, vol. 52, pp. 53-64, 2013.

[42] L. Liu, W. Cao, B. Shi, and M. Tang, "Large-scale green supplier selection approach under a q-rung interval-valued orthopair fuzzy environment," Processes, vol. 7, no. 9, p. 573, 2019. 